\title{
MEASURING MESH QUALITIES AND APPLICATION TO VARIATIONAL MESH ADAPTATION*
}

\author{
WEIZHANG HUANG ${ }^{\dagger}$
}

\begin{abstract}
The mesh assessment problem is investigated in this paper by taking into account the shape and size of elements and the solution behavior. Three elementwise mesh quality measures characterizing the shape, alignment, and adaptation features of elements are introduced according to the estimates of interpolation error developed on a general mesh. An adaptive mesh is assessed by an overall quality measure defined as a weighted Lebesgue norm of a product of the three elementwise quality measures. It is shown that the overall quality of a mesh is good if the overall mesh quality measure is small or significantly smaller than the so-called roughness measure of the solution, defined as the ratio of two Lebesgue norms of a derivative of the solution. The definition of the overall mesh quality measure comes in such a way that the measure appears in the underlying error bound as the only factor depending substantially on the mesh. As an immediate result, the task of mesh adaptation becomes to control the overall mesh quality. This idea is applied to variational mesh adaptation to develop two functionals, one new and the other related to an existing functional recently developed using the regularity and equidistribution arguments. Numerical experiments are given to demonstrate the ability of the functionals to generate adaptive meshes of good quality.
\end{abstract}

Key words. mesh quality, mesh adaptation, variational mesh adaptation, error estimate

AMS subject classifications. 65M50,65M60,65L50,65L60

DOI. $10.1137 / \mathrm{S} 1064827503429405$

1. Introduction. In the last two decades, variational mesh adaptation has received considerable attention from scientists and engineers; see [8, 9, 13, 15, 18, 19, $21,23,24,29,32]$ and the books $[14,22,28,31]$ and references therein. With a variational method, adaptive meshes are generated as images of a reference mesh under the coordinate transformation determined by a so-called adaptation functional. Such a functional is commonly designed to measure the difficulty in the numerical approximation of the physical solution. It often involves mesh properties and employs a monitor function to control mesh concentration. The development of variational mesh adaptation has so far focused on the design of the adaptation functional (e.g., see $[9,13,18,24]$ ), and there is little work on assessment of an existing mesh for a given solution. Mesh assessment is not without importance, especially since many variational methods generate a mesh of unknown quality. A good understanding of the effects of mesh qualities on the solution error can in turn help with the design of a better adaptation functional. Moreover, studies of mesh quality may lead to rigorous error analysis on adaptive meshes, which is much needed in the context of variational mesh adaptation.

Mesh assessment has been extensively studied in the context of finite elements; e.g., see the recent review paper [3] and references therein. For example, the minimum angle [36], the maximum angle [6, 20,26, 30], and the aspect ratio [11] have been widely used to characterize the shape of elements in the traditional (isotropic)

*Received by the editor, June 4, 2003; accepted for publication (in revised form) June 9, 2004; published electronically April 19, 2005.

http://www.siam.org/journals/sisc/26-5/42940.html

${ }^{\dagger}$ Department of Mathematics, the University of Kansas, Lawrence, KS 66045 (huang@math.ku. edu). This work was supported in part by the NSF under grants DMS-0074240 and DMS-0112375 and by the University of Kansas General Research Fund. Part of the work was done while the author was visiting the Department of Mathematics of the University of Strathclyde, Glasgow for a month in 2002 under an EPSRC (UK) visiting fellowship. 
error analysis. A mesh quality indicator, which takes into account both the shape of elements and the local solution behavior, is proposed by Berzins [7] for triangular and tetrahedral meshes. A so-called matching function is used by Kunert [27] to measure the correspondence of a mesh to the anisotropic feature of the solution.

In this paper we are concerned with mesh assessment and its application to variational mesh adaptation. Our objective is twofold. The first is to develop an overall mesh quality measure based on interpolation error estimates. We begin this with the derivation of interpolation error estimates for a general affine family of triangulations. According to these results, three elementwise quality measures are introduced, the geometric, alignment, and adaptation quality measures which characterize the shape of elements, the alignment of elements with the local solution feature, and the coupling between the size of elements and the solution behavior, respectively. The overall mesh quality measure is defined as a weighted Lebesgue norm of a product of the three elementwise quality measures. The definition comes in such a way that the measure appears in the underlying error bound as the only factor depending substantially on the mesh. As an immediate result, the task of mesh adaptation becomes to control the overall mesh quality. Our second objective is to apply this idea to variational mesh adaptation to develop two adaptation functionals, one new and the other related to the one developed in [18] using the regularity and equidistribution arguments. The ability of the functionals to generate adaptive meshes of good quality is demonstrated via numerical results.

In addition to its role in designing new adaptation methods, the so-defined overall mesh quality measure has several other features distinct from the existing measures [3]. First, mesh equidistribution (or adaptation) is taken into account (through the adaptation quality measure) in the definition. This is necessary for accurate assessment of adaptive meshes. Second, the definition sheds light on the effects of the aspect ratio, alignment, and adaptation mesh qualities on the overall mesh quality and therefore the interpolation error. Evidently, all of these factors have direct effects on the error. On the other hand, their effects also compensate for each other since the three elementwise measures appear in the overall quality measure as a product. As a consequence, a mesh can maintain a good overall quality when its smaller elements are worse shaped than larger elements or when well aligned elements (with the solution) are worse shaped than worse aligned elements. Finally, a mesh can be assessed using the overall mesh quality measure. It will be shown that the overall quality of a mesh is good when the quality measure is small or significantly smaller than the solution roughness measure, defined as the ratio of two Lebesgue norms of a derivative of the solution.

It is noted that our study is based on interpolation error estimates, and the results and methods developed in this paper involve derivatives of the exact solution, which typically are unavailable during the course of a numerical simulation. Fortunately, numerical experiments show that our methods work well with the numerical approximations of the solution derivatives obtained using a derivative recovery or approximation technique, such as those developed by Zienkiewicz and Zhu [34, 35], Zhang and Naga [33], and Dolejsi [12].

The paper is organized as follows. In section 2 we derive several estimates for interpolation error on a general mesh. Mesh quality measures are defined and studied in section 3. An analytical example is given in section 4 to illustrate the quality measures. In section 5, two adaptation functionals are developed based on the idea of controlling the overall mesh quality. Numerical results are presented in section 6 . Finally, section 7 contains conclusions and further remarks. 
2. Interpolation error estimates. In this section we develop several estimates for interpolation error on a general mesh.

Let $\Omega \subset \Re^{n}(n \geq 1)$ be a polyhedral physical domain. It is assumed that an affine family of triangulations $\left\{T_{h}\right\}$ is given on $\Omega$. By affine family, we mean that for each element $K$ of $T_{h} \in\left\{T_{h}\right\}$ there exists an invertible affine mapping $F_{K}: \hat{K} \rightarrow K$ such that $K=F_{K}(\hat{K})$, where $\hat{K}$ is the reference element with size $|\hat{K}|=\mathrm{O}(1)$. The norm and seminorm of Sobolev space $W^{m, p}(K)$ are denoted by $\|\cdot\|_{W^{m, p}(K)}$ and $|\cdot|_{W^{m, p}(K)}$, respectively. We will also use the scaled seminorm $\langle\cdot\rangle_{W^{m, p}(K)} \equiv(1 /|K|)^{1 / p}|\cdot|_{W^{m, p}(K)}$, which is an average.

2.1. A preliminary result. The following lemma is a standard result in the theory of interpolation on finite elements, and the interested reader is referred to, e.g., Ciarlet [11], for its proof.

LEMMA 2.1. Let $(\hat{K}, \hat{P}, \hat{\Sigma})$ be a finite element, where $\hat{K}$ is the reference element, $\hat{P}$ is a finite-dimensional linear space of functions defined on $\hat{K}$, and $\hat{\Sigma}$ is a set of degrees of freedom. Let $s$ be the greatest order of partial derivatives occurring in $\hat{\Sigma}$. For some integers $m, k$, and $l, 0 \leq m \leq l \leq k+1$, and some numbers $p, q \in[1, \infty]$, if

$$
\begin{aligned}
& W^{l, p}(\hat{K}) \hookrightarrow C^{s}(\hat{K}), \\
& W^{l, p}(\hat{K}) \hookrightarrow W^{m, q}(\hat{K}), \\
& P_{k}(\hat{K}) \subset \hat{P} \subset W^{m, q}(\hat{K}),
\end{aligned}
$$

where $P_{k}(\hat{K})$ is the space of polynomials of degree no more than $k$, then there exists a constant $C=C(\hat{K}, \hat{P}, \hat{\Sigma})$ such that, for all affine-equivalent finite elements $\left(K, P_{K}, \Sigma_{K}\right)$,

$$
\left|v-\Pi_{k, K} v\right|_{W^{m, q}(K)} \leq C\left\|\left(F_{K}^{\prime}\right)^{-1}\right\|^{m}\left|\operatorname{det}\left(F_{K}^{\prime}\right)\right|^{\frac{1}{q}}|\hat{v}|_{W^{l, p}(\hat{K})} \quad \forall v \in W^{l, p}(K),
$$

where $\Pi_{k, K}$ denotes the $P_{K}$-interpolation operator on $K$ and $\hat{v}=v \circ F_{K}$ is the composite function defined on $\hat{K}$.

It is remarked that the error bound in (2.4) is given in derivatives on $\hat{K}$. This is crucial to our study since it allows us to develop error bounds coupling mesh properties with solution derivatives on $K$. Also, (2.4) is not optimal when $m \geq 1$, but it greatly simplifies the discussion since there is no need to introduce conditions like the maximum angle condition.

It is instructive to spell out the conditions (2.1)-(2.3). By the Sobolev embedding theorem (e.g., see [1]), we have

$$
\begin{aligned}
& \left\{\begin{array}{ll}
l>\frac{n}{p}+s & \text { for } p>1 \\
l \geq n+s & \text { for } p=1
\end{array} \quad \Rightarrow W^{l, p}(\hat{K}) \hookrightarrow C^{s}(\hat{K}),\right. \\
& \left\{\begin{array}{ll}
l \geq m & \text { for } p \geq q \\
l<\frac{n}{p}+m & \text { for } \frac{1}{q}=\frac{1}{p}-\frac{l-m}{n} \\
l=\frac{n}{p}+m & \text { for } 1 \leq q<\infty
\end{array} \Rightarrow W^{l, p}(\hat{K}) \hookrightarrow W^{m, q}(\hat{K}),\right.
\end{aligned}
$$

where $n$ is the dimension of $\hat{K}$. Regarding (2.3), we note that $\hat{P}$ is often chosen as $P_{k}(\hat{K})$. If this is the case, condition (2.3) places no constraints on the parameters $m$, $k, l, p$, and $q$.

Take the widely used case of Lagrange interpolation $(s=0)$ with $p=q=2$ as an example. Condition (2.5) becomes $0 \leq m \leq l \leq k+1$ and $l>n / 2$. Thus, (2.4) holds for functions in $H^{1}(\hat{K}) \equiv W^{1,2}(\hat{K})$ in one dimension and for functions in $H^{2}(\hat{K}) \equiv W^{2,2}(\hat{K})$ in two and three dimensions. 
Hereafter we assume that the integers $m, k$, and $l$ and the numbers $p, q \in[1, \infty]$ have been chosen such that (2.4) holds. In particular, we assume that $l \geq 1,0 \leq m \leq$ $l \leq k+1$, and $q \leq p$. We use $C$ to denote the generic constant independent of $v, K$, $T_{h}$, and $N$ (the total number of the elements of $T_{h}$ ) but possibly dependent on $n, \hat{K}$, $m, k, l, p$, and $q$.

2.2. Anisotropic error estimates. We now estimate $|\hat{v}|_{W^{l, p}(\hat{K})}$ in (2.4) using derivatives on $K$. Denote by $\boldsymbol{\xi}=\left[\xi_{1}, \ldots, \xi_{n}\right]^{T}$ the coordinates for $\hat{K}$ and by $\boldsymbol{x}=$ $\left[x_{1}, \ldots, x_{n}\right]^{T}$ the local coordinates for $K$. Define the length scales of $K$ along the coordinate directions as

$$
h_{i, K}=\left(\sum_{j=1}^{n}\left|\frac{\partial x_{i}}{\partial \xi_{j}}\right|^{2}\right)^{\frac{1}{2}}, \quad i=1, \ldots, n .
$$

Let

$$
D^{\left(i_{1}, \ldots, i_{l}\right)} v=\frac{\partial^{l} v}{\partial x_{i_{1}}, \ldots, \partial x_{i_{l}}}, \quad \hat{D}^{\left(i_{1}, \ldots, i_{l}\right)} v=\frac{\partial^{l} \hat{v}}{\partial \xi_{i_{1}}, \ldots, \partial \xi_{i_{l}}} .
$$

Then, changing variables and noticing that $F_{K}^{\prime}$ is constant, we have

$$
|\hat{v}|_{W^{l, p}(\hat{K})}=\left(\operatorname{det}\left(F_{K}^{\prime}\right)^{-1} \int_{K} \sum_{i_{1}, \ldots, i_{l}}\left|\hat{D}^{\left(i_{1}, \ldots, i_{l}\right)} \hat{v}\right|^{p} d \boldsymbol{x}\right)^{\frac{1}{p}} .
$$

By the chain-rule, we get, for a given integer $t, 0 \leq t \leq l$,

$$
\begin{aligned}
& \sum_{i_{1}, \ldots, i_{l}}\left|\hat{D}^{\left(i_{1}, \ldots, i_{l}\right)} \hat{v}\right|^{p} \\
& =\sum_{i_{2}, \ldots, i_{l}} \sum_{i_{1}}\left|\sum_{j_{1}} \frac{\partial x_{j_{1}}}{\partial \xi_{i_{1}}} \hat{D}^{\left(i_{2}, \ldots, i_{l}\right)} D^{\left(j_{1}\right)} v\right|^{p} \\
& \leq C \sum_{j_{1}} h_{j_{1}, K}^{p} \sum_{i_{2}, \ldots, i_{l}}\left|\hat{D}^{\left(i_{2}, \ldots, i_{l}\right)} D^{\left(j_{1}\right)} v\right|^{p} \leq \cdots \\
& \leq C \sum_{j_{1}, \ldots, j_{l-t}} h_{j_{1}, K}^{p}, \ldots, h_{j_{l-t}, K}^{p} \sum_{i_{l-t+1}, \ldots, i_{l}}\left|\hat{D}^{\left(i_{l-t+1}, \ldots, i_{l}\right)} D^{\left(j_{1}, \ldots, j_{l-t}\right)} v\right|^{p} .
\end{aligned}
$$

2.2.1. Case $l=1$. For this case, condition (2.5) implies $s=0$ and $p>n$. Taking $t=0$ in (2.7) and using (2.4), we get

$$
\begin{aligned}
\left|v-\Pi_{k, K} v\right|_{W^{m, q}(K)} \leq & C\left\|\left(F_{K}^{\prime}\right)^{-1}\right\|^{m}\left|\operatorname{det}\left(F_{K}^{\prime}\right)\right|^{\frac{1}{q}} \\
& \times \sum_{i_{1}, \ldots, i_{l}} h_{i_{1}, K}, \ldots, h_{i_{l}, K}\left\langle D^{\left(i_{1}, \ldots, i_{l}\right)} v\right\rangle_{L^{p}(K)} .
\end{aligned}
$$

For $l=1,(2.8)$ reduces to

$$
\left|v-\Pi_{k, K} v\right|_{W^{m, q}(K)} \leq C\left\|\left(F_{K}^{\prime}\right)^{-1}\right\|^{m}\left|\operatorname{det}\left(F_{K}^{\prime}\right)\right|^{\frac{1}{q}} \sum_{i} h_{i, K}\left\langle\frac{\partial v}{\partial x_{i}}\right\rangle_{L^{p}(K)} .
$$


Note that (2.8) allows separate control of the length scales based on the derivatives in the corresponding coordinate directions. According to (2.9), for example, $h_{i, K}$ can be chosen based on the derivative $\partial v / \partial x_{i}$. This type of estimate is referred to as an anisotropic estimate in the literature. An anisotropic estimate similar to (2.8) has been developed by Apel and Dobrowolski [4] and Apel [2] .

Following [19], we derive here a different anisotropic estimate more suitable for mesh generation. For $l=1$, taking $t=1$ in (2.7) yields

$$
\begin{aligned}
\sum_{i}\left|\hat{D}^{(i)} \hat{v}\right|^{p} & =\sum_{i}\left|\sum_{j} \frac{\partial x_{j}}{\partial \xi_{i}} \frac{\partial v}{\partial x_{j}}\right|^{p}=\left(\sum_{i}\left|\left(F_{K}^{\prime} \boldsymbol{e}_{i}\right)^{T} \nabla v\right|^{p}\right) \\
& \leq C\left\|\left(F_{K}^{\prime}\right)^{T} \nabla v\right\|_{F}^{p}=C\left[\operatorname{tr}\left(\left(F_{K}^{\prime}\right)^{T} \nabla v \nabla v^{T} F_{K}^{\prime}\right)\right]^{\frac{p}{2}}
\end{aligned}
$$

where $\|\cdot\|_{F}$ is the Frobenius matrix norm and $\boldsymbol{e}_{i}$ is the $i$ th unit vector of $\Re^{n}$. By (2.4) and Jensen's inequality, we obtain

$$
\begin{aligned}
\left|v-\Pi_{k, K} v\right|_{W^{m, q}(K) \leq} & C\left\|\left(F_{K}^{\prime}\right)^{-1}\right\|^{m}\left|\operatorname{det}\left(F_{K}^{\prime}\right)\right|^{\frac{1}{q}} \\
& \times\left\langle\operatorname{tr}\left(\left(F_{K}^{\prime}\right)^{T} \nabla v \nabla v^{T} F_{K}^{\prime}\right)\right\rangle_{L^{\frac{p}{2}}(K)}^{\frac{1}{2}} .
\end{aligned}
$$

We note that when $p / 2<1,|\cdot|_{L^{p / 2}(K)}=\left[\int_{K}|\cdot|^{p / 2} d \boldsymbol{x}\right]^{2 / p}$ is no longer a norm since it does not satisfy the triangular inequality, and $L^{p / 2}(K)$ is not well defined as a normed function space. However, for simplicity we will keep using the notation $|\cdot|_{L^{p / 2}(K)}$ for the quantity $\left[\int_{K}|\cdot|^{p / 2} d \boldsymbol{x}\right]^{2 / p}$ and $\langle\cdot\rangle_{L^{p / 2}(K)}$ for the quantity $\left[(1 /|K|) \int_{K}|\cdot|^{p / 2} d \boldsymbol{x}\right]^{2 / p}$.

2.2.2. Case $\boldsymbol{l} \geq \mathbf{2}$. For $l \geq 2$, taking $t=2$ in (2.7) yields

$$
\begin{aligned}
& \sum_{i_{1}, \ldots, i_{l}}\left|\hat{D}^{\left(i_{1}, \ldots, i_{l}\right)} \hat{v}\right|^{p} \\
& \quad \leq C \sum_{j_{1}, \ldots, j_{l-2}} h_{j_{1}, K}^{p}, \ldots, h_{j_{l-2}, K}^{p} \sum_{i_{l-1}, i_{l}}\left|\sum_{j_{l-1}, j_{l}} \frac{\partial x_{j_{l-1}}}{\partial \xi_{i_{l-1}}} \frac{\partial x_{j_{l}}}{\partial \xi_{i_{l}}} \frac{\partial^{2}\left(D^{\left(j_{1}, \ldots, j_{l-2}\right)} v\right)}{\partial x_{j_{l-1}} \partial x_{j_{l}}}\right|^{p} \\
& \leq C \sum_{j_{1}, \ldots, j_{l-2}} h_{j_{1}, K}^{p}, \ldots, h_{j_{l-2}, K}^{p} \sum_{i_{l-1}, i_{l}}\left|\left(F_{K}^{\prime} \boldsymbol{e}_{i_{l-1}}\right)^{T} H\left(D^{\left(j_{1}, \ldots, j_{l-2}\right)} v\right)\left(F_{K}^{\prime} \boldsymbol{e}_{i_{l}}\right)\right|^{p}
\end{aligned}
$$

where $H\left(D^{\left(j_{1}, \ldots, j_{l-2}\right)} v\right)$ denotes the Hessian of $D^{\left(j_{1}, \ldots, j_{l-2}\right)} v$. Let

$$
\left|H\left(D^{\left(j_{1}, \ldots, j_{l-2}\right)} v\right)\right|=Q \operatorname{diag}\left(\left|\lambda_{1}\right|, \ldots,\left|\lambda_{n}\right|\right) Q^{T},
$$

where $Q$ is the orthogonal matrix consisting of the (normalized) eigenvectors and the $\lambda_{i}$ 's are the eigenvalues of $H$. It is easy to show (see [19])

$$
\left|\boldsymbol{a}^{T} H \boldsymbol{b}\right| \leq \frac{1}{2}\left(\boldsymbol{a}^{T}|H| \boldsymbol{a}+\boldsymbol{b}^{T}|H| \boldsymbol{b}\right) \quad \forall \boldsymbol{a}, \boldsymbol{b} \in \Re^{n} .
$$

Combining this with (2.11) yields

$$
\begin{aligned}
\sum_{i_{1}, \ldots, i_{l}}\left|\hat{D}^{\left(i_{1}, \ldots, i_{l}\right)} \hat{v}\right|^{p} & \leq C \sum_{j_{3}, \ldots, j_{l}} h_{j_{3}, K}^{p}, \ldots, h_{j_{l}, K}^{p} \sum_{i}\left(\left(F_{K}^{\prime} \boldsymbol{e}_{i}\right)^{T}\left|H\left(D^{\left(j_{3}, \ldots, j_{l}\right)} v\right)\right|\left(F_{K}^{\prime} \boldsymbol{e}_{i}\right)\right)^{p} \\
& \leq C \sum_{j_{3}, \ldots, j_{l}} h_{j_{3}, K}^{p}, \ldots, h_{j_{l}, K}^{p}\left[\operatorname{tr}\left(\left(F_{K}^{\prime}\right)^{T}\left|H\left(D^{\left(j_{3}, \ldots, j_{l}\right)} v\right)\right| F_{K}^{\prime}\right)\right]^{p} .
\end{aligned}
$$


By (2.4) and $h_{i_{1}, K}, \ldots, h_{i_{l-2}, K} \leq C\left\|F_{K}^{\prime}\right\|^{l-2}$, we have

$$
\begin{aligned}
\left|v-\Pi_{k, K} v\right|_{W^{m, q}(K)} \leq & C\left\|\left(F_{K}^{\prime}\right)^{-1}\right\|^{m}\left\|F_{K}^{\prime}\right\|^{l-2}\left|\operatorname{det}\left(F_{K}^{\prime}\right)\right|^{\frac{1}{q}} \\
& \times\left\langle\operatorname{tr}\left(\left(F_{K}^{\prime}\right)^{T}\left|H\left(D^{l-2} v\right)\right| F_{K}^{\prime}\right)\right\rangle_{L^{p}(K)},
\end{aligned}
$$

where

$$
\left|H\left(D^{l-2} v\right)\right| \equiv \sum_{i_{1}, \ldots, i_{l-2}}\left|H\left(D^{\left(i_{1}, \ldots, i_{l-2}\right)} v\right)\right| .
$$

The estimates (2.10) and (2.13) are of anisotropic type. To explain this, we take (2.13) with $l=2$ as an example. Using (2.12) and letting $Q=\left[\boldsymbol{q}_{1}, \ldots, \boldsymbol{q}_{n}\right]$, we get

$$
\operatorname{tr}\left(\left(F_{K}^{\prime}\right)^{T}|H(v)| F_{K}^{\prime}\right)=\sum_{i}\left|\lambda_{i}\right| \cdot\left\|\left(F_{K}^{\prime}\right)^{T} \boldsymbol{q}_{i}\right\|^{2}
$$

Thus, the length scale of $K$ in the direction $\boldsymbol{q}_{i},\left\|\left(F_{K}^{\prime}\right)^{T} \boldsymbol{q}_{i}\right\|$, can be chosen (in the $L^{p}$ sense) based on $\sqrt{\left|\lambda_{i}\right|}$ during the course of mesh generation.

It is interesting to point out that (2.10) and (2.13) are independent of the choice of the local coordinate system on $K$. This is because the terms involved in the bounds, such as the norm, determinant, and trace of $F_{K}^{\prime}$, are coordinate-independent.

3. Mesh quality measures. We define and study in this section the mesh quality measures according to the interpolation error estimates obtained in the previous section. Our basic tools are the following lemmas. The interested reader is referred to Hardy, Littlewood, and Pólya [16] and Kober [25] for their proofs.

LEMma 3.1 (the arithmetic-mean geometric-mean inequality and its refined version). For any $r$ positive numbers $a_{1}, \ldots, a_{r}$,

$$
\left(\prod_{i=1}^{r} a_{i}\right)^{\frac{1}{r}} \leq \frac{1}{r} \sum_{i=1}^{r} a_{i}
$$

with equality if and only if $a_{1}=\cdots=a_{r}$. The inequality can be refined as

$$
\frac{1}{r(r-1)} \sum_{i<j}\left(\sqrt{a_{i}}-\sqrt{a_{j}}\right)^{2} \leq \frac{1}{r} \sum_{i} a_{i}-\left(\prod_{i} a_{i}\right)^{\frac{1}{r}} \leq \frac{1}{r} \sum_{i<j}\left(\sqrt{a_{i}}-\sqrt{a_{j}}\right)^{2} .
$$

Lemma 3.2. Given a weight function $w(\boldsymbol{x})$ with $\int_{\Omega} w d \boldsymbol{x}=1$ on a bounded domain $\Omega \subset \Re^{n}$, let

$$
M_{r}(f)=\left(\int_{\Omega} w|f|^{r} d \boldsymbol{x}\right)^{1 / r}
$$

for arbitrary function $f$ and real number $r$, with the limits that $M_{0}(f)=\exp \left(\int_{\Omega} w\right.$ $\log |f| d \boldsymbol{x})$ (geometric mean), $M_{+\infty}=\max |f|$, and $M_{-\infty}=\min |f|$. Then

$$
M_{r}(f) \leq M_{s}(f) \quad \text { for }-\infty \leq r<s \leq+\infty,
$$

with equality if and only if (a) $M_{r}(f)=M_{s}(f)=+\infty$, (b) $M_{r}(f)=M_{s}(f)=0$, or (c) $f \equiv$ constant. 
3.1. Geometric quality measure. The geometric quality measure which characterizes the shape of $K$ can be defined in a number of ways (e.g., see [3]), such as using the maximum angle, the minimum angle, or the aspect ratio. Our objective is to define a measure which is easy to compute, suitable for mesh adaptation, and consistent with the bounds given in (2.8) and (2.13). Motivated by Lemma 3.1 and the fact that the trace and the determinant of a matrix are equal to the sum and the product of its eigenvalues, respectively, we define the geometric quality measure in terms of $F_{K}^{\prime}$ and its inverse $\left(F_{K}^{\prime}\right)^{-1}$ as

$$
\begin{aligned}
& Q_{\text {geo }}(K)=\left[\frac{\operatorname{tr}\left(\left(F_{K}^{\prime}\right)^{T} F_{K}^{\prime}\right)}{n \operatorname{det}\left(\left(F_{K}^{\prime}\right)^{T} F_{K}^{\prime}\right)^{\frac{1}{n}}}\right]^{\frac{n}{2(n-1)}}=\left[\frac{\left\|F_{K}^{\prime}\right\|_{F}}{\sqrt{n} \operatorname{det}\left(F_{K}^{\prime}\right)^{\frac{1}{n}}}\right]^{\frac{n}{n-1}} \\
& \hat{Q}_{\mathrm{geo}}(K)=\left[\frac{\operatorname{tr}\left(\left(F_{K}^{\prime}\right)^{-1}\left(F_{K}^{\prime}\right)^{-T}\right)}{n \operatorname{det}\left(\left(F_{K}^{\prime}\right)^{-1}\left(F_{K}^{\prime}\right)^{-T}\right)^{\frac{1}{n}}}\right]^{\frac{n}{2(n-1)}}=\left[\frac{\left\|\left(F_{K}^{\prime}\right)^{-1}\right\|_{F}}{\sqrt{n} \operatorname{det}\left(F_{K}^{\prime}\right)^{-\frac{1}{n}}}\right]^{\frac{n}{n-1}}
\end{aligned}
$$

Note that $Q_{\text {geo }}(K)=\hat{Q}_{\text {geo }}(K)$ when $n=2$. Obviously, these measures are easy to compute. Moreover, by (3.2) it is not difficult to show (cf. the proof of Lemma 4.1 in [19]) that

$$
\begin{aligned}
& 1 \leq Q_{\mathrm{geo}}(K) \leq \frac{\mu_{\mathrm{max}}}{\mu_{\mathrm{min}}} \leq\left[\sqrt{n(n-1)\left(Q_{\mathrm{geo}}^{\frac{2(n-1)}{n}}(K)-1\right)}+1\right]^{2 n}, \\
& 1 \leq \hat{Q}_{\mathrm{geo}}(K) \leq \frac{\mu_{\mathrm{max}}}{\mu_{\mathrm{min}}} \leq\left[\sqrt{n(n-1)\left(\hat{Q}_{\mathrm{geo}}^{\frac{2(n-1)}{n}}(K)-1\right)}+1\right]^{2 n},
\end{aligned}
$$

where $\mu_{\min }$ and $\mu_{\max }$ are the minimum and maximum singular values, respectively, of the Jacobian matrix $F_{K}^{\prime}$. Since the singular values are the lengths of the semiaxes of the hyperellipsoid $\left\{\boldsymbol{x} \mid \boldsymbol{x}=F_{K}^{\prime} \boldsymbol{\xi},\|\boldsymbol{\xi}\|=1\right\}$, we conclude by (3.6) and (3.7) that $Q_{\text {geo }}(K)$ and $\hat{Q}_{\text {geo }}(K)$ are equivalent to $\mu_{\max } / \mu_{\min }$, which in turn is equivalent to the aspect ratio of $K$. Moreover, $Q_{\text {geo }}(K)=1$ or $\hat{Q}_{\text {geo }}(K)=1$ implies that $\mu_{\max } / \mu_{\min }=1$ or $K$ is equilateral.

3.2. Case $\boldsymbol{l}=\mathbf{1}$. The estimate (2.10) can be rewritten as

$$
\begin{aligned}
\left|v-\Pi_{k, K} v\right|_{W^{m, q}(K) \leq} \leq & C \hat{Q}_{\mathrm{geo}}^{\frac{m(n-1)}{n}}(K)\left|\operatorname{det}\left(F_{K}^{\prime}\right)\right|^{\frac{1}{q}-\frac{m}{n}} \\
& \times\left\langle\operatorname{tr}\left(\left(F_{K}^{\prime}\right)^{T} \nabla v \nabla v^{T} F_{K}^{\prime}\right)\right\rangle_{L^{\frac{p}{2}}(K)}^{\frac{1}{2}} .
\end{aligned}
$$

3.2.1. The alignment quality measure. As for the geometric quality measure, the definition of the alignment quality measure is also motivated by Lemma 3.1 but associated with $\left\langle\operatorname{tr}\left(\left(F_{K}^{\prime}\right)^{T} \nabla v \nabla v^{T} F_{K}^{\prime}\right)\right\rangle_{L^{p / 2}(K)}$ in (3.8). Since $\left(F_{K}^{\prime}\right)^{T} \nabla v \nabla v^{T} F_{K}^{\prime}$ is only semi-positive definite, Lemma 3.1 cannot be directly applied to its eigenvalues. To avoid this difficulty, we regularize the expression with a flooring parameter $\alpha_{h, 1}>0$, which will be defined in (3.16). (The subscript 1 stands for $l=1$.) Replace $\operatorname{tr}\left(\left(F_{K}^{\prime}\right)^{T} \nabla v \nabla v^{T} F_{K}^{\prime}\right)$ with

$$
\operatorname{tr}\left(\left(F_{K}^{\prime}\right)^{T}\left[\alpha_{h, 1} I+\nabla v \nabla v^{T}\right] F_{K}^{\prime}\right)=\alpha_{h, 1} \operatorname{tr}\left(\left(F_{K}^{\prime}\right)^{T}\left[I+\frac{1}{\alpha_{h, 1}} \nabla v \nabla v^{T}\right] F_{K}^{\prime}\right) .
$$


By Lemma 3.1, we have

$$
\begin{aligned}
\operatorname{tr}\left(\left(F_{K}^{\prime}\right)^{T}\left[I+\frac{1}{\alpha_{h, 1}} \nabla v \nabla v^{T}\right] F_{K}^{\prime}\right) & \geq n \operatorname{det}\left(\left(F_{K}^{\prime}\right)^{T}\left[I+\frac{1}{\alpha_{h, 1}} \nabla v \nabla v^{T}\right] F_{K}^{\prime}\right)^{\frac{1}{n}} \\
& =n \operatorname{det}\left(F_{K}^{\prime}\right)^{\frac{2}{n}}\left(1+\frac{1}{\alpha_{h, 1}}\|\nabla v\|^{2}\right)^{\frac{1}{n}} .
\end{aligned}
$$

Thus we define the alignment quality measure as

$$
Q_{\mathrm{ali}, 1}(K)=\left[\frac{\left\langle\operatorname{tr}\left(\left(F_{K}^{\prime}\right)^{T}\left[I+\frac{1}{\alpha_{h, 1}} \nabla v \nabla v^{T}\right] F_{K}^{\prime}\right)\right\rangle_{L^{\frac{p}{2}}(K)}}{n \operatorname{det}\left(F_{K}^{\prime}\right)^{\frac{2}{n}}\left\langle 1+\frac{1}{\alpha_{h, 1}}\|\nabla v\|^{2}\right\rangle_{L^{\frac{1}{2 n}}}^{\frac{1}{2 n}}(K)}\right]^{\frac{n}{2(n-1)}} .
$$

To see the geometric meaning of $Q_{\mathrm{ali}, 1}$, we denote the normalized eigensystem of $I+\left(1 / \alpha_{h, 1}\right) \nabla v \nabla v^{T}$ by $\left(\lambda_{i}, \boldsymbol{q}_{i}\right), i=1, \ldots, n$. Using $\prod_{i}\left\|\left(F_{K}^{\prime}\right)^{T} \boldsymbol{q}_{i}\right\|^{2} \geq \operatorname{det}\left(F_{K}^{\prime}\right)^{2}$ and an equation similar to $(2.14)$, we have

$$
\frac{\operatorname{tr}\left(\left(F_{K}^{\prime}\right)^{T}\left[I+\frac{1}{\alpha_{h, 1}} \nabla v \nabla v^{T}\right] F_{K}^{\prime}\right)}{n \operatorname{det}\left(\left(F_{K}^{\prime}\right)^{T}\left[I+\frac{1}{\alpha_{h, 1}} \nabla v \nabla v^{T}\right] F_{K}^{\prime}\right)^{\frac{1}{n}}} \geq \frac{\sum_{i} \lambda_{i}\left\|\left(F_{K}^{\prime}\right)^{T} \boldsymbol{q}_{i}\right\|^{2}}{n\left(\prod_{i} \lambda_{i}\left\|\left(F_{K}^{\prime}\right)^{T} \boldsymbol{q}_{i}\right\|^{2}\right)^{\frac{1}{n}}} \geq 1
$$

Thus, $Q_{\text {ali }, 1}(K)=1$ implies that $\sqrt{\lambda_{i}}\left\|\left(F_{K}^{\prime}\right)^{T} \boldsymbol{q}_{i}\right\|, i=1, \ldots, n$, are equal to a constant (in the $L^{p / 2}(K)$ sense). In other words, the length scale of $K$ in the direction $\boldsymbol{q}_{i}$, $\left\|\left(F_{K}^{\prime}\right)^{T} \boldsymbol{q}_{i}\right\|(i=1, \ldots, n)$, is reversely proportional to $\sqrt{\lambda_{i}}$. In this sense, the shape of $K$ is aligned with the eigensystem of $I+\left(1 / \alpha_{h, 1}\right) \nabla v \nabla v^{T}$, or, loosely speaking, with the geometry of $v$. On the other hand, it is easy to see from (3.2) that the farther $\sqrt{\lambda_{i}}\left\|\left(F_{K}^{\prime}\right)^{T} \boldsymbol{q}_{i}\right\|, i=1, \ldots, n$, differ from each other, the larger $Q_{\text {ali, } 1}(K)$ will be. This indicates that $Q_{\mathrm{ali}, 1}(K)$ measures how far the quantities $\sqrt{\lambda_{i}}\left\|\left(F_{K}^{\prime}\right)^{T} \boldsymbol{q}_{i}\right\|, i=1, \ldots, n$, are from being constant, or how well (the shape of) element $K$ is aligned with the geometry of $v$.

3.2.2. Equidistribution and the adaptation quality measure. The definition of the adaptation quality measure is associated with the size of $K,\left|\operatorname{det}\left(F_{K}^{\prime}\right)\right|$, and based on the well-known equidistribution principle [10]. For the moment, let us assume that a so-called adaptation function $\rho=\rho_{K, 1}>0$ is given. (Function $\rho_{K, 1}$ will be defined in (3.14) for the current case.) Then a multidimensional generalization of the equidistribution principle is given by

$$
|K| \rho_{K, 1}=\frac{\sigma_{h, 1}}{N} \quad \text { with } \quad \sigma_{h, 1}=\sum_{K}|K| \rho_{K, 1},
$$

where $N$ is the total number of the elements. It implies that the size of $K$ should be small for large $\rho_{K}$ and vice versa. We define the adaptation quality measure as

$$
Q_{\mathrm{adp}, 1}(K)=\frac{N|K| \rho_{K, 1}}{\sigma_{h, 1}} .
$$

$Q_{\text {adp }, 1}(K)$ measures how well the mesh is equidistributing according to $\rho_{K, 1}$. In fact, it is not difficult to show that $\max _{K} Q_{\mathrm{adp}, 1}(K) \geq 1$, and the equality holds if and only if the equidistribution relation is satisfied exactly. The farther the mesh is from satisfying (3.10), the larger $\max _{K} Q_{\mathrm{adp}, 1}(K)$ will be. 
3.2.3. Choice of $\boldsymbol{\rho}_{\boldsymbol{K}, \mathbf{1}}$ and $\boldsymbol{\alpha}_{\boldsymbol{h , 1}}$. Using $Q_{\mathrm{ali}, 1}(K), Q_{\mathrm{adp}, 1}(K)$, and $\left|\operatorname{det}\left(F_{K}^{\prime}\right)\right| \sim$ $|K|$, we get from (3.8)

$$
\begin{aligned}
& \sum_{K}\left|v-\Pi_{k, K} v\right|_{W^{m, q}(K)}^{q} \leq C \alpha_{h, 1}^{\frac{q}{2}}\left(\frac{\sigma_{h, 1}}{N}\right)^{\frac{q(1-m)}{n}} \sum_{K}|K| \\
& (3.12) \times\left(\hat{Q}_{\mathrm{geo}^{n}}^{\frac{m(n-1)}{n}}(K) Q_{\mathrm{ali}, 1}^{\frac{n-1}{n}}(K) Q_{\mathrm{adp}, 1}^{\frac{1-m}{n}}(K)\right)^{q} \rho_{K, 1}^{-\frac{q(1-m)}{n}}\left\langle 1+\frac{1}{\alpha_{h, 1}}\|\nabla v\|^{2}\right\rangle_{L^{\frac{p}{2 n}}(K)}^{\frac{q}{2 n}}
\end{aligned}
$$

Following [19], we choose $\rho_{K, 1}$ such that

$$
\rho_{K, 1}^{-\frac{q(1-m)}{n}}\left\langle 1+\frac{1}{\alpha_{h, 1}}\|\nabla v\|^{2}\right\rangle_{L^{\frac{p}{2 n}}(K)}^{\frac{q}{2 n}}=\rho_{K, 1},
$$

which yields

$$
\rho_{K, 1}=\left\langle 1+\frac{1}{\alpha_{h, 1}}\|\nabla v\|^{2}\right\rangle_{L \frac{p}{2 n}(K)}^{\frac{\gamma}{2}}
$$

where $\gamma$ is defined as

$$
\gamma=\frac{q}{n+q(l-m)}
$$

(Recall that $l=1$ for the current case.)

One may notice from (3.14) that the flooring parameter plays a role in weighting the effect of $v$ on the adaptation function. For this reason, $\alpha_{h, 1}$ is often referred to as the (adaptation) intensity parameter in the literature. Evidently, $\alpha_{h, 1}$ cannot be chosen too large or too small. Huang and Sun [19] suggest that $\alpha_{h, 1}$ be chosen such that (i) it has the same dimension as $\|\nabla v\|^{2}$ and (ii) $\sigma_{h, 1} \equiv \sum_{K}|K| \rho_{K, 1} \leq C$ for some constant $C$. This gives

$$
\alpha_{h, 1}=\left[\frac{1}{|\Omega|} \sum_{K}|K|\langle\|\nabla v\|\rangle_{L^{\frac{p}{n}}(K)}^{\gamma}\right]^{\frac{2}{\gamma}} .
$$

Then, using (3.13) and (3.16), the estimate (3.12) becomes

$$
\left[\sum_{K}\left|v-\Pi_{k, K} v\right|_{W^{m, q}(K)}^{q}\right]^{\frac{1}{q}} \leq C N^{-\frac{1-m}{n}}\left[\sum_{K}|K|\langle\|\nabla v\|\rangle_{L^{\frac{p}{n}}(K)}^{\gamma}\right]^{\frac{1}{\gamma}} Q_{\mathrm{mesh}, h, 1},
$$

where

$$
\begin{aligned}
& Q_{\text {mesh }, h, 1} \\
& \equiv\left[\frac{1}{\left(\sum_{K}|K| \rho_{K, 1}\right)} \sum_{K}|K| \rho_{K, 1}\left(\hat{Q}_{\text {geo }^{n}}^{\frac{m(n-1)}{n}}(K) Q_{\mathrm{ali}, 1}^{\frac{n-1}{n}}(K) Q_{\mathrm{adp}, 1}^{\frac{1-m}{n}}(K)\right)^{q}\right]^{\frac{1}{q}} .
\end{aligned}
$$


$Q_{\text {mesh }, h, 1}$ is the overall quality measure of the mesh. It takes into account the shape (through $\hat{Q}_{\mathrm{geo}}(K)$ and $Q_{\mathrm{ali}, 1}(K)$ ) and the size (through $Q_{\mathrm{adp}, 1}(K)$ ) of elements and the solution behavior (through $Q_{\mathrm{ali}, 1}(K)$ and $Q_{\mathrm{adp}, 1}(K)$ ). In particular, equidistribution (or adaptation) is incorporated (through $Q_{\mathrm{adp}, 1}(K)$ ) into the definition. The inclusion of equidistribution renders $Q_{\text {mesh } h, 1}$ more suitable for measuring the overall quality of adaptive meshes.

Two observations can be made from (3.17) and (3.18). The first is that (3.17) reveals the relation between the mesh qualities and the interpolation error. Apparently, the geometric, alignment, and adaptation mesh qualities directly affect the interpolation error. On the other hand, since they appear in $Q_{\text {mesh }, h, 1}$ as a product, their effects are not independent but instead compensate for each other. As a consequence, the mesh can maintain a good overall quality when small elements (with smaller $Q_{\mathrm{adp}, 1}$ ) are shaped worse, in the sense of having larger $\hat{Q}_{\text {geo }}$ than large elements, or wellaligned elements (with smaller $Q_{\mathrm{ali}, 1}$ ) are worse shaped than worse aligned elements. The other observation comes from

$$
\langle\|\nabla v\|\rangle_{L^{\gamma}(\Omega)} \leq\left[\frac{1}{|\Omega|} \sum_{K}|K|\langle\|\nabla v\|\rangle_{L^{\frac{p}{n}}(K)}^{\gamma}\right]^{\frac{1}{\gamma}} \leq\langle\|\nabla v\|\rangle_{L^{\frac{p}{n}}(\Omega)},
$$

which follows from Lemmas 3.1 and 3.2 and the fact that $\gamma \leq p / n$. In other words, the summation term on the right-hand side of (3.17) is bounded below and above by meshindependent numbers. Thus, we can conclude that $Q_{\text {mesh }, h, 1}$ has been defined in such a way that it appears in the error bound as the only factor depending substantially on the mesh. As an immediate result, the task of mesh adaptation becomes to control $Q_{\text {mesh }, h, 1}$ during the course of mesh adaptation.

3.2.4. Mesh assessment. An adaptive mesh is assessed by comparing the solution error thereon to its counterpart on a uniform mesh with the same number of elements. An error bound on a uniform mesh is obtained from (2.4), (2.7), and the assumption $q \leq p$ as, for any $l \geq 1$,

$$
\left[\sum_{K}\left|v-\Pi_{k, K} v\right|_{W^{m, q}(K)}^{q}\right]^{\frac{1}{q}} \leq C N^{-\frac{l-m}{n}}|v|_{W^{l, p}(\Omega)},
$$

where we have used $|K|=|\Omega| / N$ and $Q_{\text {geo }}(K)=\hat{Q}_{\text {geo }}(K)=1$ for the uniform mesh. By requiring that the error bound given in (3.17) be much smaller than that given in (3.20), neglecting the constants, and writing in a dimensionless form, we get

$$
Q_{\text {mesh }, h, 1} \ll Q_{\text {soln }, h, 1},
$$

where

$$
Q_{\mathrm{soln}, h, 1}=\frac{\langle\|\nabla v\|\rangle_{L^{p}(\Omega)}}{\sqrt{\alpha_{h, 1}}} .
$$

To see the physical meaning of $Q_{\mathrm{soln}, h, 1}$, from (3.16) and (3.19) we have

$$
1 \leq \frac{\langle\|\nabla v\|\rangle_{L^{p}(\Omega)}}{\langle\|\nabla v\|\rangle_{L^{\frac{p}{n}}(\Omega)}} \leq Q_{\mathrm{soln}, h, 1} \leq \frac{\langle\|\nabla v\|\rangle_{L^{p}(\Omega)}}{\langle\|\nabla v\|\rangle_{L^{\gamma}(\Omega)}} \leq \frac{\max _{\boldsymbol{x}}\|\nabla v\|(\boldsymbol{x})}{\min _{\boldsymbol{x}}\|\nabla v\|(\boldsymbol{x})},
$$


where we have used Lemma 3.2 in the first and last inequalities. We claim that $Q_{\text {soln }, h, 1}$ indicates how well $v$ can be approximated by interpolation on a uniform mesh. (For this reason, we will call $Q_{\text {soln }, h, 1}$ the roughness measure of $v$.) To see this we note that how far its gradient is from being constant is equivalent to how well $v$ can be approximated by interpolation on a uniform mesh. Meanwhile, Lemma 3.2 and (3.23) imply that $Q_{\operatorname{soln}, h, 1}=1$ when $\nabla v$ is constant and $Q_{\operatorname{soln}, h, 1}$ is large if $\nabla v$ is far from being constant.

We now can see from (3.21) that the overall mesh quality is good if $Q_{\text {mesh, } h, 1} \ll$ $Q_{\text {soln }, h, 1}$ for a tough solution (i.e., a solution with large $\left.Q_{\operatorname{soln}, h, 1}\right)$. On the other hand, when the solution is smooth, an adaptive mesh will lead to very little improvement in accuracy over a uniform mesh. Hence, the overall quality of a mesh is good if

$$
\begin{array}{ll}
Q_{\text {mesh }, h, 1}=O(1) & \text { if } Q_{\mathrm{soln}, h, 1}=O(1), \\
Q_{\text {mesh }, h, 1} \ll Q_{\mathrm{soln}, h, 1} & \text { if } Q_{\mathrm{soln}, h, 1} \gg 1 .
\end{array}
$$

In other words, a mesh has a good overall quality if $Q_{\mathrm{mesh}, h, 1}$ is small or significantly smaller than $Q_{\mathrm{soln}, h, 1}$.

3.2.5. Continuous form. It is often instructive to formulate mesh quality measures and error bounds in a continuous form. Let the coordinate transformation be $\boldsymbol{x}=\boldsymbol{x}(\boldsymbol{\xi}): \Omega_{c} \rightarrow \Omega$, where $\Omega_{c}$ is the computational or logic domain. Denote the Jacobian matrix and its determinant by

$$
\boldsymbol{J}=\frac{\partial \boldsymbol{x}}{\partial \boldsymbol{\xi}}, \quad J=\operatorname{det}(\boldsymbol{J}) .
$$

Then the continuous form of the equidistribution principle (3.10) reads as

$$
J \rho_{1}(\boldsymbol{x})=\frac{\sigma_{1}}{\left|\Omega_{c}\right|} \quad \text { with } \quad \sigma_{1}=\int_{\Omega} \rho_{1} d \boldsymbol{x} .
$$

Moreover, we have

$$
\begin{aligned}
& Q_{\text {geo }}(\boldsymbol{x})=\left[\frac{\operatorname{tr}\left(\boldsymbol{J}^{T} \boldsymbol{J}\right)}{n J^{\frac{2}{n}}}\right]^{\frac{n}{2(n-1)}}=\left[\frac{\|\boldsymbol{J}\|_{F}}{\sqrt{n} J^{\frac{1}{n}}}\right]^{\frac{n}{n-1}}, \\
& \hat{Q}_{\mathrm{geo}}(\boldsymbol{x})=\left[\frac{\operatorname{tr}\left(\boldsymbol{J}^{-1} \boldsymbol{J}^{-T}\right)}{n J^{-\frac{2}{n}}}\right]^{\frac{n}{2(n-1)}}=\left[\frac{\left\|\boldsymbol{J}^{-1}\right\|_{F}}{\sqrt{n} J^{-\frac{1}{n}}}\right]^{\frac{n}{n-1}}, \\
& \alpha_{1}=\left[\frac{1}{|\Omega|} \int_{\Omega}\|\nabla v\|^{\gamma} d \boldsymbol{x}\right]^{\frac{2}{\gamma}}, \\
& \rho_{1}(\boldsymbol{x})=\left[1+\frac{1}{\alpha_{1}}\|\nabla v\|^{2}\right]^{\frac{\gamma}{2}}, \\
& Q_{\mathrm{ali}, 1}(\boldsymbol{x})=\left[\frac{\operatorname{tr}\left(\boldsymbol{J}^{T}\left[I+\frac{1}{\alpha_{1}} \nabla v \nabla v^{T}\right] \boldsymbol{J}\right)}{n J^{\frac{2}{n}}\left(1+\frac{1}{\alpha_{1}}\|\nabla v\|^{2}\right)^{\frac{1}{n}}}\right]^{\frac{n}{2(n-1)}}, \\
& Q_{\mathrm{adp}, 1}(\boldsymbol{x})=\frac{\rho_{1}(\boldsymbol{x}) J\left|\Omega_{c}\right|}{\sigma_{1}} \text { with } \sigma_{1}=\int_{\Omega}^{\rho_{1}(\boldsymbol{x}) d \boldsymbol{x},}
\end{aligned}
$$




$$
\begin{aligned}
& {\left[\sum_{K}\left|v-\Pi_{k, K} v\right|_{W^{m, q}(K)}^{q}\right]^{\frac{1}{q}} \lesssim C N^{-\frac{(1-m)}{n}} Q_{\mathrm{mesh}, 1}|v|_{W^{1, \gamma}(\Omega)},} \\
& Q_{\mathrm{mesh}, 1}=\left[\frac{1}{\left(\int_{\Omega} \rho_{1} d \boldsymbol{x}\right)} \int_{\Omega} \rho_{1}\left(\hat{Q}_{\mathrm{geo}^{n}}^{\frac{m(n-1)}{n}} Q_{\mathrm{ali}, 1}^{\frac{n-1}{n}} Q_{\mathrm{adp}, 1}^{\frac{1-m}{n}}\right)^{q} d \boldsymbol{x}\right]^{\frac{1}{q}}, \\
& Q_{\mathrm{soln}, 1}=\frac{\langle\|\nabla v\|\rangle_{L^{p}(\Omega)}}{\langle\|\nabla v\|\rangle_{L^{\gamma}(\Omega)}}=\frac{\langle\|\nabla v\|\rangle_{L^{p}(\Omega)}}{\sqrt{\alpha_{1}}},
\end{aligned}
$$

where $\gamma$ is defined in (3.15) and $\lesssim$ is used in (3.32) to indicate that the estimate holds only asymptotically.

3.3. Case $\boldsymbol{l} \geq 2$. For this case, the quality measures and the adaptation function can be defined as in the previous subsection according to estimate (2.13). Indeed, we have

$$
\begin{aligned}
Q_{\mathrm{adp}, 2}(K) & =\frac{N|K| \rho_{K, 2}}{\sigma_{h, 2}} \text { with } \sigma_{h, 2}=\sum_{K}|K| \rho_{K, 2}, \\
Q_{\mathrm{ali}, 2}(K) & =\left[\frac{\left\langle\operatorname{tr}\left(\left(F_{K}^{\prime}\right)^{T}\left[I+\frac{1}{\alpha_{h, 2}}\left|H\left(D^{l-2} v\right)\right|\right] F_{K}^{\prime}\right)\right\rangle_{L^{p}(K)}}{n \operatorname{det}\left(F_{K}^{\prime}\right)^{\frac{2}{n}}\left\langle\operatorname{det}\left(I+\frac{1}{\alpha_{h, 2}}\left|H\left(D^{l-2} v\right)\right|\right)\right\rangle_{L^{\frac{1}{n}}(K)}^{\frac{1}{n}}}\right]^{\frac{n}{2(n-1)}}, \\
\rho_{K, 2} & =\left\langle\operatorname{det}\left(I+\frac{1}{\alpha_{h, 2}}\left|H\left(D^{l-2} v\right)\right|\right)\right\rangle_{L^{\frac{p}{n}}(K)}^{\gamma},
\end{aligned}
$$

where $\gamma$ is defined in (3.15). For the current case we cannot have an explicit expression for $\alpha_{h, 2}$ since it cannot be separated from the determinant in (3.37). Nevertheless, we can define $\alpha_{h, 2}$ implicitly through

$$
\sigma_{h, 2} \equiv \sum_{K}|K| \rho_{K, 2}=2^{\frac{n \gamma(p-1)}{p}+\max \left\{0, \frac{n \gamma}{p}-1\right\}}|\Omega| .
$$

The calculation of $\alpha_{h, 2}$ is neither difficult nor time-consuming. (We simply use the bisection method in our computations.) It can readily be shown that

$$
\alpha_{h, 2} \leq \frac{1}{n}\left[\frac{1}{|\Omega|} \sum_{K}|K|\left\langle\operatorname{tr}\left(\left|H\left(D^{l-2} v\right)\right|\right)\right\rangle_{L^{p}(K)}^{n \gamma}\right]^{\frac{1}{n \gamma}} .
$$

The global bound on the interpolation error is given by

$$
\left[\sum_{K}\left|v-\Pi_{k, K} v\right|_{W^{m, q}(K)}^{q}\right]^{\frac{1}{q}} \leq C N^{-\frac{l-m}{n}} \alpha_{h, 2} Q_{\text {mesh }, h, 2},
$$

where the overall mesh quality measure and the solution roughness are defined as

$$
\begin{aligned}
& Q_{\mathrm{mesh}, h, 2}=\left[\frac{1}{\left(\sum_{K}|K| \rho_{K, 2}\right)}\right. \\
& \left.\times \sum_{K}|K| \rho_{K, 2}\left(\hat{Q}_{\text {geo }^{n}}^{\frac{m(n-1)}{n}}(K) Q_{\mathrm{geo}^{\frac{(l-2)(n-1)}{n}}}(K) Q_{\mathrm{ali}, 2}^{\frac{2(n-1)}{n}}(K) Q_{\mathrm{adp}, 2}^{\frac{l-m}{n}}(K)\right)^{q}\right]^{\frac{1}{q}},
\end{aligned}
$$




$$
Q_{\text {soln }, h, 2}=\frac{\langle v\rangle_{W^{l, p}(\Omega)}}{\alpha_{h, 2}} .
$$

$Q_{\text {soln, } h, 2}$ measures how well the function $v$ can be approximated by piecewise polynomials of degree no more than $k$ on a uniform mesh.

In the continuous form, the above formulas read as

$$
\begin{aligned}
& \rho_{2}(\boldsymbol{x})=\operatorname{det}\left(I+\frac{1}{\alpha_{2}}\left|H\left(D^{l-2} v\right)\right|\right)^{\gamma}, \\
& \sigma_{2} \equiv \int_{\Omega} \rho_{2}(\boldsymbol{x}) d \boldsymbol{x}=2^{\frac{n \gamma(p-1)}{p}}+\max \left\{0, \frac{n \gamma}{p}-1\right\}|\Omega|, \\
& \alpha_{2} \leq \frac{1}{n}\left[\frac{1}{|\Omega|} \int_{\Omega} \operatorname{tr}\left(\left|H\left(D^{l-2} v\right)\right|\right)^{n \gamma} d \boldsymbol{x}\right]^{\frac{1}{n \gamma}}, \\
& Q_{\mathrm{ali}, 2}(\boldsymbol{x})=\left[\frac{\operatorname{tr}\left(\boldsymbol{J}^{T}\left[I+\frac{1}{\alpha_{2}}\left|H\left(D^{l-2} v\right)\right|\right] \boldsymbol{J}\right)}{n J^{\frac{2}{n}} \operatorname{det}\left(I+\frac{1}{\alpha_{2}}\left|H\left(D^{l-2} v\right)\right|\right)^{\frac{1}{n}}}\right]^{\frac{n}{2(n-1)}}, \\
& Q_{\mathrm{adp}, 2}(\boldsymbol{x})=\frac{\rho_{2}(\boldsymbol{x}) J\left|\Omega_{c}\right|}{\sigma_{2}}, \\
& {\left[\sum_{K}\left|v-\Pi_{k, K} v\right|_{W^{m, q}(K)}^{q}\right]^{\frac{1}{q}} \lesssim C N^{-\frac{(l-m)}{n}} \alpha_{2} Q_{\mathrm{mesh}, 2},} \\
& \left.Q_{\mathrm{mesh}, 2}=\left[\frac{1}{\left(\int_{\Omega} \rho_{2} d \boldsymbol{x}\right)} \int_{\Omega}^{\rho_{2}\left(\hat{Q}_{\mathrm{geo}}^{\frac{m(n-1)}{n}}\right.} Q_{\mathrm{geo}}^{\frac{(l-2)(n-1)}{n}} Q_{\mathrm{ali}, 2}^{\frac{2(n-1)}{n}} Q_{\mathrm{adp}, 2}^{\frac{l-m}{n}}\right)^{q} d \boldsymbol{x}\right]^{\frac{1}{q}}, \\
& Q_{\mathrm{soln}, 2}=\frac{\langle v\rangle_{W^{l, p}(\Omega)}}{\alpha_{2}} .
\end{aligned}
$$

Note that the observations made in section 3.2 .3 for case $l=1$ can also be made from the above formulas for the current case $l \geq 2$.

To conclude this section, we remark that the ratio $Q_{\text {mesh }, h} / Q_{\mathrm{soln}, h}$ plays a similar role in the error bounds as the matching function used by Kunert [27]. However, it is reported in [27] that the matching function can be small or large for misadapted meshes.

4. An analytical example. To better understand the quality measures defined in the previous section, we consider a two-dimensional example:

$$
v(x, y)=e^{-\frac{x}{\epsilon}}+e^{-\frac{y}{\epsilon}}, \quad(x, y) \in \Omega=(0,1) \times(0,1),
$$

which exhibits boundary layers near $x=0$ and $y=0$ when $\epsilon \ll 1$. This function is a solution of the singularly perturbed partial differential equation $-\epsilon^{2} \Delta v+v=$ 0 . Function (4.1) is selected as our example because it is simple enough to work analytically and it is known (e.g., see [5]) that a Shishkin-type mesh can resolve the boundary layers.

We consider the linear interpolation $(k=1$ and $l=2)$ on a Shishkin-type rectangular mesh as shown in Figure 4.1. The interpolation error is measured in the seminorm of $H^{1}(\Omega)$ (i.e., $p=q=2$ and $m=1$ ). The mesh parameters are given by

$$
a=a_{0} \epsilon|\ln \epsilon|, \quad h=\frac{1-a}{M}, \quad \bar{h}=\frac{a}{M},
$$




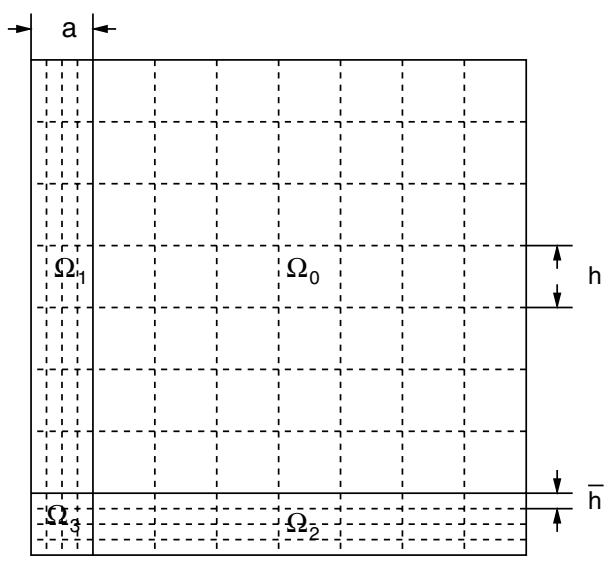

FIG. 4.1. Sketch of a Shishkin-type rectangular mesh.

for some given integer $M>0$. For simplicity, we use the continuous formulas in the following analysis. We choose $a_{0} \geq 2$ so that the second derivatives of $v$ remain bounded in $\Omega_{0}$ as $\epsilon \rightarrow 0$.

For this example, $\gamma=1 / 2$ and $|H(v)|=\operatorname{diag}\left(\left|v_{x x}\right|,\left|v_{y y}\right|\right)$. Equation (3.44) reads as

$$
\int_{\Omega} \operatorname{det}\left(I+\frac{1}{\alpha_{2}}|H(v)|\right)^{\frac{1}{2}} d x d y=2^{\frac{3}{2}}|\Omega|,
$$

which leads to

$$
\alpha_{2}=O(1)
$$

The adaptation function (3.43) becomes

$$
\rho_{2}(x, y)=\operatorname{det}\left(I+\frac{1}{\alpha_{2}}|H(v)|\right)^{\frac{1}{2}}= \begin{cases}O(1) & \text { in } \Omega_{0}, \\ O\left(\epsilon^{-1}\right) & \text { in } \Omega_{1}, \Omega_{2}, \\ O\left(\epsilon^{-2}\right) & \text { in } \Omega_{3} .\end{cases}
$$

The alignment quality measure (3.46) reads as

$$
Q_{\mathrm{ali}, 2}(x, y)=\frac{h_{x}^{2}\left(1+\alpha_{2}^{-1}\left|v_{x x}\right|\right)+h_{y}^{2}\left(1+\alpha_{2}^{-1}\left|v_{y y}\right|\right)}{2 h_{x} h_{y} \rho_{2}(x, y)},
$$

where $h_{x}$ and $h_{y}$ are the length scales of elements in the $x$ and $y$ directions, respectively. The quality measures and the estimate for interpolation error are listed in Table 4.1. For comparison, we also list the results obtained with (4.2) and (4.3) on a uniform mesh.

We observe that the alignment and adaptation qualities are improved, whereas the geometric quality deteriorates from a uniform mesh to a Shishkin-type one. Particularly, the geometric quality of the elements in the regions $\Omega_{1}$ and $\Omega_{2}$ becomes very bad for the Shishkin-type mesh. Nevertheless, due to the improvements in alignment and adaptation, the Shishkin-type mesh has a better overall quality and results in a smaller interpolation error. For both meshes, $Q_{\text {mesh,2 }} \rightarrow \infty$ as $\epsilon \rightarrow 0$. However, $Q_{\text {mesh,2 }} \ll Q_{\text {soln,2 }}$ for the Shishkin-type mesh and $Q_{\text {mesh }, 2}=O\left(Q_{\text {soln, } 2}\right)$ for the uniform one. According to the analysis given in section 3 (cf. (3.24)), the overall quality 
TABLE 4.1

Quality measures and interpolation error for Shishkin-type and uniform meshes for example (4.1). In the table, $|e|_{H^{1}(\Omega)}=\left(\sum_{K}\left|v-\Pi_{k, K} v\right|_{H^{1}(K)}^{2}\right)^{\frac{1}{2}}$. It can be shown that $Q_{\text {soln }, 2}=O\left(\epsilon^{-3 / 2}\right)$.

\begin{tabular}{c|l|l|l|l|l|l}
\hline \hline & \multicolumn{3}{|c|}{ Uniform mesh } & \multicolumn{3}{c}{ Shishkin-type mesh } \\
\hline \hline & $\Omega_{0}$ & $\Omega_{1}, \Omega_{2}$ & $\Omega_{3}$ & $\Omega_{0}$ & $\Omega_{1}, \Omega_{2}$ & $\Omega_{3}$ \\
\cline { 2 - 7 }$Q_{\text {geo }}(x, y)$ & $O(1)$ & $O(1)$ & $O(1)$ & $O(1)$ & $O\left(\epsilon^{-1}|\ln \epsilon|^{-1}\right)$ & $O(1)$ \\
$Q_{\text {ali }, 2}(x, y)$ & $O(1)$ & $O\left(\epsilon^{-1}\right)$ & $O(1)$ & $O(1)$ & $O(|\ln \epsilon|)$ & $O(1)$ \\
$Q_{\text {adp }, 2}(x, y)$ & $O(1)$ & $O\left(\epsilon^{-1}\right)$ & $O\left(\epsilon^{-2}\right)$ & $O(1)$ & $O(|\ln \epsilon|)$ & $O\left(|\ln \epsilon|^{2}\right)$ \\
\hline \hline$Q_{\text {mesh }, 2}$ & \multicolumn{3}{|c|}{$O\left(\epsilon^{-\frac{3}{2}}|\ln \epsilon|\right)$} & \multicolumn{3}{c}{$O\left(\epsilon^{\left.-\frac{1}{2}|\ln \epsilon|^{\frac{3}{2}}\right)}\right.$} \\
\hline$Q_{\text {mesh, } 2 / Q_{\text {soln }, 2}}$ & \multicolumn{3}{|c|}{$\mathrm{O}(1)$} & \multicolumn{3}{c}{$O(\epsilon)$} \\
\hline$|e|_{H^{1}(\Omega)}$ & \multicolumn{3}{|c|}{$O\left(M^{-1} \epsilon^{-\frac{3}{2}}|\ln \epsilon|\right)$} & \multicolumn{3}{c}{$O\left(M^{-1} \epsilon^{-\frac{1}{2}}|\ln \epsilon|^{\frac{3}{2}}\right)$} \\
\hline \hline
\end{tabular}

of the Shishkin-type mesh is good, whereas that of the uniform mesh is bad. This is not surprising since the uniform mesh is obviously misaligned with function (4.1) in the regions $\Omega_{1}$ and $\Omega_{2}$ and misadapted in all regions but $\Omega_{0}$. It is worth pointing out that $Q_{\text {mesh,2 }} / Q_{\text {soln,2 }}=O(1)$ and thus is small for this misadapted (uniform) mesh. This means that the ratio, which plays a similar role as the matching function in [27] (see the remark at the end of section 3), is not an appropriate measure of the overall mesh quality.

5. Adaptation functionals for variational mesh adaptation. We have seen in section 3 that the task for mesh adaptation is to control the overall mesh quality. In this section we apply this idea to variational mesh generation to develop several adaptation functionals.

5.1. Case $\boldsymbol{l}=1$. We first consider the overall mesh quality measure (3.18). By Jensen's inequality, we have, for a given $s \in(0,1]$,

$$
\begin{aligned}
{\left[\sigma_{h, 1} Q_{\mathrm{mesh}, h, 1}^{q}\right]^{s} } & =\left[\sum_{K}|K| \rho_{K, 1}\left(\hat{Q}_{\mathrm{geo}}^{\frac{m(n-1)}{n}}(K) Q_{\mathrm{ali}, 1}^{\frac{n-1}{n}}(K) Q_{\mathrm{adp}, 1}^{\frac{1-m}{n}}(K)\right)^{q}\right]^{s} \\
& \leq \sum_{K}\left(|K| \rho_{K, 1}\right)^{s}\left(\hat{Q}_{\mathrm{geo}^{\frac{m(n-1)}{n}}}^{n}(K) Q_{\mathrm{ali}, 1}^{\frac{n-1}{n}}(K) Q_{\mathrm{adp}, 1}^{\frac{1-m}{n}}(K)\right)^{s q} \\
& =\sum_{K}|K| \rho_{K, 1}\left(|K| \rho_{K, 1}\right)^{s-1}\left(\hat{Q}_{\mathrm{geo}^{n}}^{\frac{m(n-1)}{n}}(K) Q_{\mathrm{ali}, 1}^{\frac{n-1}{n}}(K) Q_{\mathrm{adp}, 1}^{\frac{1-m}{n}}(K)\right)^{s q} \\
(5.1) \quad & \sim\left(\frac{\sigma_{1}}{N}\right)^{s-1} \int_{\Omega} \rho_{1}(\boldsymbol{x}) Q_{\mathrm{adp}, 1}^{s-1}(\boldsymbol{x})\left(\hat{Q}_{\operatorname{geo}^{\frac{m(n-1)}{n}}}(\boldsymbol{x}) Q_{\mathrm{ali}, 1}^{\frac{n-1}{n}}(\boldsymbol{x}) Q_{\mathrm{adp}, 1}^{\frac{1-m}{n}}(\boldsymbol{x})\right)^{s q} d \boldsymbol{x} .
\end{aligned}
$$

Bearing the equidistribution relation (3.10) or (3.25) in mind, we rewrite the integral on the right-hand side as

$$
\begin{aligned}
\int_{\Omega} \rho_{1}(\boldsymbol{x}) Q_{\mathrm{adp}, 1}^{s-1}(\boldsymbol{x})\left(\hat{Q}_{\mathrm{geo}}^{\frac{m(n-1)}{n}}(\boldsymbol{x}) Q_{\mathrm{ali}, 1}^{\frac{n-1}{n}}(\boldsymbol{x}) Q_{\mathrm{adp}, 1}^{\frac{1-m}{n}}(\boldsymbol{x})\right)^{s q} d \boldsymbol{x} \\
=C \int_{\Omega} \rho_{1}\left[\frac{1}{n} \sum_{i}\left(\nabla \xi^{i}\right)^{T} M_{1}^{-1} \nabla \xi^{i}\right]^{\frac{s q m}{2}} \\
\quad \times\left[\frac{1}{n} \sum_{i}\left(\nabla \xi^{i}\right)^{T} G_{1}^{-1} \nabla \xi^{i}\right]^{\frac{s q(n-1)}{2}}\left(J \rho_{1}\right)^{s q+s-1} d \boldsymbol{x},
\end{aligned}
$$


where we have used (3.29) and $M_{1}$ and $G_{1}$ are defined as

$$
\begin{aligned}
& M_{1}=\rho_{1}^{\frac{2}{n}} I, \\
& G_{1}=\left(1+\frac{1}{\alpha_{1}}\|\nabla v\|^{2}\right)^{\frac{\gamma(m q-n)}{n q}}\left[I+\frac{1}{\alpha_{1}} \nabla v \nabla v^{T}\right] .
\end{aligned}
$$

It is straightforward to use the right-hand-side term of (5.1) as the adaptation functional for mesh generation. Unfortunately, its highly nonlinear and nonconvex nature makes it difficult to find its extremals. (Indeed, our limited experience shows that the mesh can easily become singular in computation.) For this reason, we seek alternatives as follows. For simplicity we consider only the widely used case $m=0$ for the current situation $l=1$.

5.1.1. The bound-based approach. In this approach, the adaptation functional is constructed using a bound for (5.2). In fact, (5.2) yields

$$
\begin{aligned}
& \int_{\Omega} \rho_{1}\left[\frac{1}{n} \sum_{i}\left(\nabla \xi^{i}\right)^{T} G_{1}^{-1} \nabla \xi^{i}\right]^{\frac{s q(n-1)}{2}}\left(J \rho_{1}\right)^{s q+s-1} d \boldsymbol{x} \\
& \leq \frac{1}{2 w} \int_{\Omega} \rho_{1}\left[\frac{1}{n} \sum_{i}\left(\nabla \xi^{i}\right)^{T} G_{1}^{-1} \nabla \xi^{i}\right]^{s q(n-1)} d \boldsymbol{x}+\frac{w}{2} \int_{\Omega} \rho_{1}\left(J \rho_{1}\right)^{2 s q+2 s-2} d \boldsymbol{x},
\end{aligned}
$$

where the weight $w$ is used to balance the first and second terms on the right-hand side. By assuming that the mesh is well equidistributed (and thus $J \rho_{1} \approx \sigma_{1} /\left|\Omega_{c}\right|$ ), the dimension of the integrands can be found as

$$
\begin{gathered}
\frac{1}{w}\left[\frac{1}{n} \sum_{i}\left(\nabla \xi^{i}\right)^{T} G_{1}^{-1} \nabla \xi^{i}\right]^{s q(n-1)} \sim \frac{1}{w}\left(J \rho_{1}\right)^{-\frac{2 s q(n-1)}{n}} \sim \frac{1}{w}\left(\frac{\sigma_{1}}{\left|\Omega_{c}\right|}\right)^{-\frac{2 s q(n-1)}{n}}, \\
w\left(J \rho_{1}\right)^{2 s q+2 s-2} \sim w\left(\frac{\sigma_{1}}{\left|\Omega_{c}\right|}\right)^{2 s q+2 s-2}
\end{gathered}
$$

Setting these equal, we get

$$
w=\left(\frac{\sigma_{1}}{\left|\Omega_{c}\right|}\right)^{-\frac{s q(n-1)}{n}-(s q+s-1)} .
$$

The adaptation functional is then defined as

$$
\begin{aligned}
I[\boldsymbol{\xi}]= & \int_{\Omega} \rho_{1}\left[\frac{1}{n} \sum_{i}\left(\nabla \xi^{i}\right)^{T} G_{1}^{-1} \nabla \xi^{i}\right]^{s q(n-1)} d \boldsymbol{x} \\
& +\left(\frac{\sigma_{1}}{\left|\Omega_{c}\right|}\right)^{-\frac{2 s q(n-1)}{n}-(2 s q+2 s-2)} \int_{\Omega} \rho_{1}\left(J \rho_{1}\right)^{2 s l q+2 s-2} d \boldsymbol{x} .
\end{aligned}
$$

By the same argument used in [18] (and by Lemmas 3.1 and 3.2), it can be shown that the first integral, which is convex when $s q(n-1) \geq 1$, is related to the alignment quality requirement, while the second integral, obviously nonconvex, is associated with the equidistribution requirement. The geometrical meaning of the second integral can also 
be seen from the fact (cf. Lemma 3.2) that a minimizer of functional $\int_{\Omega} \rho_{1}\left(J \rho_{1}\right)^{s} d \boldsymbol{x}$ for any given number $s \in \Re \backslash[-1,0]$ satisfies the equidistribution relation $J \rho_{1}=$ constant.

The choice of $s \in(0,1]$ is subject to the conditions $s q(n-1) \geq 1$ and $2 s q+2 s-2>$ 0 in order to keep the convexity of the first integral and the equidistribution tendency of the second one. In the meantime, it is desirable from the computational point of view to make the indices in the integrals as small as possible. Based on these considerations, we choose $s=2 /(q+1)$ and obtain

$$
\begin{aligned}
I_{\mathrm{bnd}, 1}[\boldsymbol{\xi}]= & \int_{\Omega} \rho_{1}\left[\frac{1}{n} \sum_{i}\left(\nabla \xi^{i}\right)^{T} G_{1}^{-1} \nabla \xi^{i}\right]^{\frac{2 q(n-1)}{q+1}} d \boldsymbol{x} \\
& +\left(\frac{\sigma_{1}}{\left|\Omega_{c}\right|}\right)^{-\frac{4 q(n-1)}{n(q+1)}-2} \int_{\Omega} \rho_{1}\left(J \rho_{1}\right)^{2} d \boldsymbol{x}
\end{aligned}
$$

5.1.2. The dimensional balancing approach. The goal of this approach is to develop a functional with dimensionally balanced terms. We have from (5.2)

$$
\begin{aligned}
& \int_{\Omega} \rho_{1}\left[\frac{1}{n} \sum_{i}\left(\nabla \xi^{i}\right)^{T} G_{1}^{-1} \nabla \xi^{i}\right]^{\frac{s q(n-1)}{2}}\left(J \rho_{1}\right)^{s q+s-1} d \boldsymbol{x} \\
& \leq \int_{\Omega} \rho_{1}\left[\frac{1}{n} \sum_{i}\left(\nabla \xi^{i}\right)^{T} G_{1}^{-1} \nabla \xi^{i}\right]^{\frac{s q(n-1)}{2}} d \boldsymbol{x} \cdot\left[\max _{\boldsymbol{x}}\left(J \rho_{1}\right)\right]^{s q+s-1} \\
& \leq C\left[\int_{\Omega} \rho_{1}\left(\frac{1}{n} \sum_{i}\left(\nabla \xi^{i}\right)^{T} G_{1}^{-1} \nabla \xi^{i}\right)^{s q(n-1)} d \boldsymbol{x}\right]^{\frac{1}{2}} \cdot\left[\max _{\boldsymbol{x}}\left(J \rho_{1}\right)\right]^{s q+s-1} .
\end{aligned}
$$

Note that minimizing $\max \boldsymbol{x}\left(J \rho_{1}\right)$ is equivalent to minimizing $\int \rho_{1}\left(J \rho_{1}\right)^{s} d \boldsymbol{x}$ for $s \in$ $\Re \backslash[-1,0]$ in the sense that all of their minimizers satisfy the equidistribution relation $J \rho_{1}=$ constant; cf. Lemma 3.2. For this reason, we define the adaptation functional as

$$
I[\boldsymbol{\xi}]=\theta \int_{\Omega} \rho_{1}\left[\frac{1}{n} \sum_{i}\left(\nabla \xi^{i}\right)^{T} G_{1}^{-1} \nabla \xi^{i}\right]^{s q(n-1)} d \boldsymbol{x}+(1-\theta) \int_{\Omega} \rho_{1}\left(J \rho_{1}\right)^{-\frac{2 s q(n-1)}{n}} d \boldsymbol{x},
$$

where $\theta \in[0,1]$ is a weight. The dimensional homogeneity of the terms in the functional can be easily verified by observing that $\sum_{i}\left(\nabla \xi^{i}\right)^{T} G_{1}^{-1} \nabla \xi^{i}$ and $\left(J \rho_{1}\right)^{-2 / n}$ have the same dimension. The first term is simply the integral on the right-hand side of the last inequality of (5.6), while the second is associated with equidistribution. By taking $s=n /(q(n-1))$, we obtain

$$
I_{\mathrm{bal}, 1}[\boldsymbol{\xi}]=\theta \int_{\Omega} \rho_{1}\left[\frac{1}{n} \sum_{i}\left(\nabla \xi^{i}\right)^{T} G_{1}^{-1} \nabla \xi^{i}\right]^{n} d \boldsymbol{x}+(1-\theta) \int_{\Omega} \rho_{1}\left(J \rho_{1}\right)^{-2} d \boldsymbol{x} .
$$

This gives exactly the same functional developed in [18] for the case $(l, m)=(1,0)$. 
5.2. Case $\boldsymbol{l} \geq 2$. For this case, we have, for $s \in(0,1]$,

$$
\begin{aligned}
{\left[\sigma_{h, 2} Q_{\mathrm{mesh}, h, 2}^{q}\right]^{s} \lesssim } & C N^{1-s} \int_{\Omega} \rho_{2}\left[\frac{1}{n} \sum_{i}\left(\nabla \xi^{i}\right)^{T} M_{2}^{-1} \nabla \xi^{i}\right]^{\frac{s q(m+(l-2)(n-1))}{2}} \\
& \times\left[\frac{1}{n} \sum_{i}\left(\nabla \xi^{i}\right)^{T} G_{2}^{-1} \nabla \xi^{i}\right]^{s q(n-1)}\left(J \rho_{2}\right)^{s l q+s-1} d \boldsymbol{x},
\end{aligned}
$$

where

$$
\begin{aligned}
& M_{2}=\rho_{2}^{\frac{2}{n}} I, \\
& G_{2}=\operatorname{det}\left(I+\frac{1}{\alpha_{2}}\left|H\left(D^{l-2} v\right)\right|\right)^{\frac{\gamma(2 q-n-q(l-m))}{n q}}\left[I+\frac{1}{\alpha_{2}}\left|H\left(D^{l-2} v\right)\right|\right] .
\end{aligned}
$$

The adaptation functional can be developed as in the previous subsection for general $l$ and $m$. For simplicity, we consider here only the commonly used case $l=2$.

5.2.1. The bound-based approach. We take $s=2 /(2 q+1)$ to get

$$
\begin{aligned}
I_{\mathrm{bnd}, 2}[\boldsymbol{\xi}]= & \int_{\Omega} \rho_{2}\left[\frac{1}{n} \sum_{i}\left(\nabla \xi^{i}\right)^{T} G_{2}^{-1} \nabla \xi^{i}\right]^{\frac{4 q(n-1)}{2 q+1}} d \boldsymbol{x} \\
& +\left(\frac{\sigma_{2}}{\left|\Omega_{c}\right|}\right)^{-\frac{4 q(n-1)}{n(2 q+1)}-2} \int_{\Omega} \rho_{2}\left(J \rho_{2}\right)^{2} d \boldsymbol{x}
\end{aligned}
$$

for $m=0$ and

$$
\begin{aligned}
I_{\mathrm{bnd}, 2}[\boldsymbol{\xi}]= & \int_{\Omega} \rho_{2}\left[\frac{1}{n} \sum_{i}\left(\nabla \xi^{i}\right)^{T} M_{2}^{-1} \nabla \xi^{i}\right]^{\frac{3 q}{2 q+1}} d \boldsymbol{x} \\
& +\int_{\Omega} \rho_{2}\left[\frac{1}{n} \sum_{i}\left(\nabla \xi^{i}\right)^{T} G_{2}^{-1} \nabla \xi^{i}\right]^{\frac{6 q(n-1)}{2 q+1}} d \boldsymbol{x} \\
& +\left(\frac{\sigma_{2}}{\left|\Omega_{c}\right|}\right)^{-\frac{12 q(n-1)}{n(2 q+1)}-3} \int_{\Omega} \rho_{2}\left(J \rho_{2}\right)^{3} d \boldsymbol{x}
\end{aligned}
$$

for $m=1$.

5.2.2. The dimensional balancing approach. Taking $s=n /(q(n-1))$, we get

$$
I_{\mathrm{bal}, 2}[\boldsymbol{\xi}]=\theta \int_{\Omega} \rho_{2}\left[\frac{1}{n} \sum_{i}\left(\nabla \xi^{i}\right)^{T} G_{2}^{-1} \nabla \xi^{i}\right]^{n} d \boldsymbol{x}+(1-\theta) \int_{\Omega} \rho_{2}\left(J \rho_{2}\right)^{-2} d \boldsymbol{x}
$$

for $m=0$ and

$$
\begin{aligned}
I_{\mathrm{bal}, 2}[\boldsymbol{\xi}]= & \frac{\theta}{2} \int_{\Omega} \rho_{2}\left[\frac{1}{n} \sum_{i}\left(\nabla \xi^{i}\right)^{T} M_{2}^{-1} \nabla \xi^{i}\right]^{\frac{n}{n-1}} d \boldsymbol{x} \\
& +\frac{\theta}{2} \int_{\Omega} \rho_{2}\left[\frac{1}{n} \sum_{i}\left(\nabla \xi^{i}\right)^{T} G_{2}^{-1} \nabla \xi^{i}\right]^{2 n} d \boldsymbol{x}+(1-\theta) \int_{\Omega} \rho_{2}\left(J \rho_{2}\right)^{-4} d \boldsymbol{x}
\end{aligned}
$$

for $m=1$. 
Functionals (5.11) and (5.13) for the case $m=1$ involve three terms, corresponding to the mesh quality requirements of geometry, alignment, and adaptation. Neglecting the first term, the dimensional balancing functional (5.13) gives the same functional developed in [18] based on the regularity and equidistribution considerations.

5.3. Remarks. The functionals developed in this section are similar to those in [18]. To save space we opt not to list their Euler-Lagrange equations here. The reader is referred to $[17,18]$ for their derivations.

Having the roles of independent and dependent variables interchanged, the EulerLagrange equation can be solved for adaptive meshes. A key step in this solution procedure is to compute the adaptation function $\left(\rho_{1}\right.$ or $\left.\rho_{2}\right)$ and the monitor functions $\left(\left(M_{1}, G_{1}\right)\right.$ or $\left.\left(M_{2}, G_{2}\right)\right)$. One may notice from $(5.3),(5.4),(5.8)$, and (5.9) that $\rho$, $M$, and $G$ involve derivatives of the solution which are typically unavailable in a numerical simulation. Fortunately, numerical experiments (also see the numerical results presented in the next section) show that our functionals work well with the numerical approximations of these derivatives obtained using a gradient recovery or approximation technique, such as those developed by Zienkeiwicz and Zhu [34, 35], Zhang and Naga [33], and Dolejsi [12].

6. Numerical results. We present in this section some two-dimensional numerical results to demonstrate the ability of the adaptation functionals developed in the previous section to generate adaptive meshes of good quality. We consider here two examples of given functions, one exhibiting mild and the other having strong anisotropic features. Without loss of generality, we consider the linear interpolation ( $k=1$ and $l=2)$, with the error being measured in the seminorm of $H^{1}(m=1$ and $p=q=2)$.

Adaptive meshes are obtained by first interchanging the roles of dependent and independent variables in the Euler-Lagrange equation of the adaptation functionals and then solving the resulting mesh equation through the so-called moving mesh PDE approach. The interested reader is referred to [17] for the implementation detail of the approach. We would like to point out that the mesh equation should ideally be discretized on a triangular computational mesh since linear interpolation is under consideration. For convenience we instead choose to discretize it on a rectangular computational mesh via finite differences and produce quadrilateral adaptive meshes (as shown in Figures 6.1 and 6.3). The needed triangular meshes (not shown in this work) can be obtained by partitioning each quadrilateral element into two triangular elements by drawing one of the diagonal lines, or into four by drawing both lines. Indeed, the linear interpolation error presented in this section is computed on a triangular mesh obtained by drawing the northeast diagonal line in each quadrilateral element of a quadrilateral mesh.

The convergent mesh is obtained when the root-mean-square norm of the residual in the mesh equations is less than $10^{-3}$. All computations start with a uniform mesh, and $\theta=0.1$ is used for the dimensional balancing functional. The boundary points are fixed for the first example but adapted through a one-dimensional mesh equation $[17]$ in the second example.

As mentioned in section 5.3, solution derivatives are needed in the computation of the adaptation function (3.43) and the monitor functions (5.8) and (5.9). Although in our examples the solution is available analytically, we have not taken this advantage in our computations. Instead, we have chosen to use the numerical approximations of the solution derivatives obtained using a local, quadratic least squares fitting polynomial 

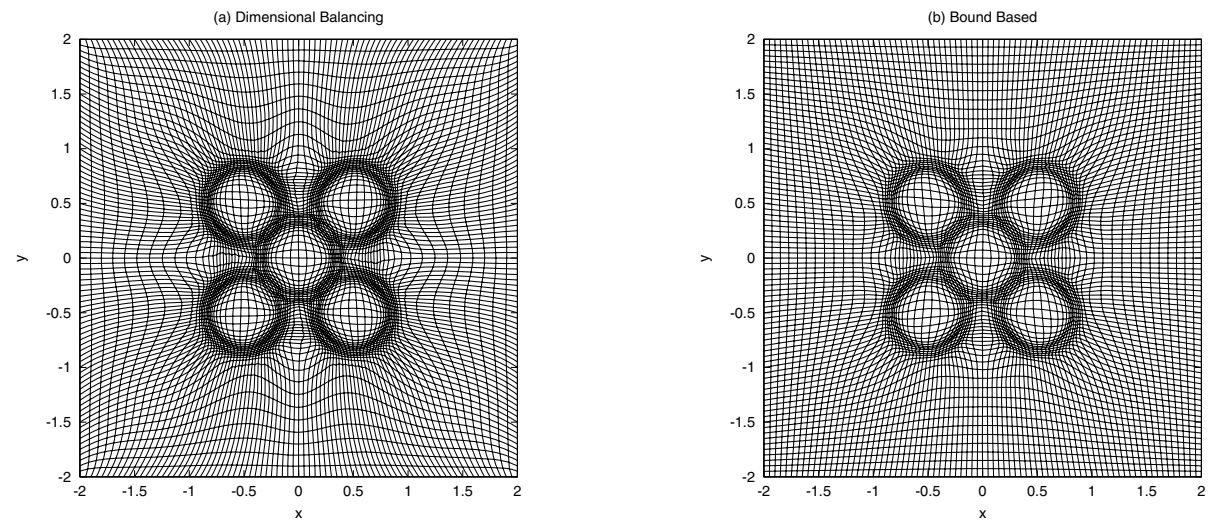

FIG. 6.1. Adaptive meshes of size $81 \times 81$ are obtained for Example 6.1 with the bound-based and dimensional balancing functionals.

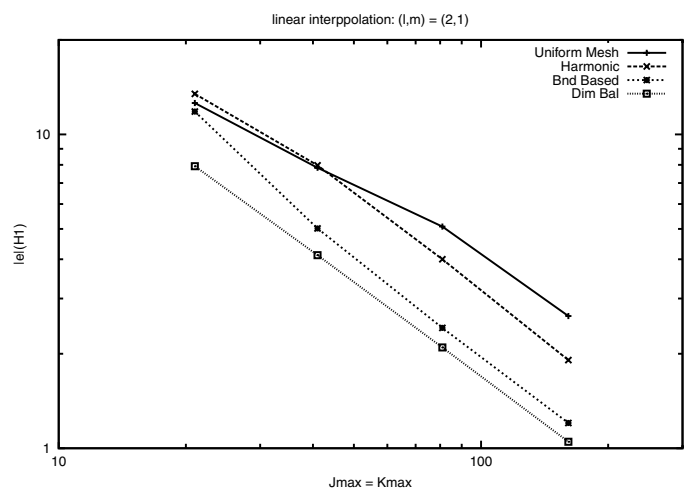

FIG. 6.2. The linear interpolation error $|e|_{H^{1}(\Omega)}$ is plotted as a function of the total number of points in one of the coordinate directions for Example 6.1.

based on neighboring nodal values of the solution [33]. This approach is more realistic and works for other problems such as the numerical solution of partial differential equations where nodal values of a computed solution are always available.

Example 6.1. The first example is

$$
v(x, y)=\sum_{i=0}^{4} \tanh \left[30\left(\left(x-x_{i}\right)^{2}+\left(y-y_{i}\right)^{2}-\frac{1}{8}\right)\right]
$$

defined on $\Omega=(-2,2) \times(-2,2)$, where $\left(x_{0}, y_{0}\right)=(0,0),\left(x_{1}, y_{1}\right)=(-0.5,-0.5)$, $\left(x_{2}, y_{2}\right)=(-0.5,0.5),\left(x_{3}, y_{3}\right)=(0.5,0.5)$, and $\left(x_{4}, y_{4}\right)=(0.5,-0.5)$. Figure 6.1 shows typical adaptive meshes obtained with the bound-based and dimensional balancing functionals. It can be seen that the mesh points are concentrated in the correct regions. There is no significant difference between the results obtained with the two functionals. This can also be observed from Figure 6.2 which shows the $H^{1}$ seminorm of the linear interpolation error against the total number of mesh points in one of the coordinate directions. Moreover, the error $|e|_{H^{1}(\Omega)}$ converges at a first order rate in $J \max$ (note: the total number of elements $N=J \max ^{2}$ ) for all cases, confirming the theoretical prediction made in section 3. For comparison, the errors are also plotted in Figure 6.2 for a uniform mesh and an adaptive mesh generated using the 
TABLE 6.1

Qualities of meshes obtained for Example 6.1 with the bound-based (Bnd-based) and dimensional balancing (Dim bal) functionals.

\begin{tabular}{l|c|c|c|c|cc}
\hline \hline Method & $J \max$ & $\left\|Q_{\text {geo }}\right\|_{\infty}$ & $\left\|Q_{\text {ali, } 2}\right\|_{\infty}$ & $\left\|Q_{\text {adp }, 2}\right\|_{\infty}$ & $Q_{\text {mesh }, 2}$ & $Q_{\text {soln }, 2}$ \\
\hline \hline Dim bal & 21 & 2.02 & 2.47 & 1.92 & 1.17 & 15.5 \\
& 41 & 2.24 & 5.34 & 2.28 & 1.24 & 15.5 \\
& 81 & 2.44 & 5.14 & 2.60 & 1.31 & 15.5 \\
& 161 & 2.84 & 4.90 & 1.96 & 1.36 & 15.5 \\
\hline \hline Bnd-based & 21 & 1.25 & 1.99 & 3.46 & 1.47 & 15.5 \\
& 41 & 1.99 & 5.15 & 3.22 & 1.49 & 15.5 \\
& 81 & 2.28 & 5.76 & 2.81 & 1.51 & 15.5 \\
& 161 & 2.93 & 5.82 & 2.14 & 1.52 & 15.5 \\
\hline \hline
\end{tabular}

harmonic map method [13] with monitor function $G_{2}$ defined in (5.9). These errors are considerably larger than those resulting from the adaptive meshes generated by the bound-based and dimensional balancing functionals.

In Table 6.1 we list the qualities of the adaptive meshes. For all cases, the geometric, alignment, and equidistribution mesh qualities measured in the maximum norm remain relatively small. Moreover, $\left\|Q_{\text {geo }}\right\|_{\infty}<3$ means that the elements are close to being equilateral. This is consistent with the fact that the function (6.1) does not have a strong anisotropic feature. Furthermore, $Q_{\text {mesh }, 2} \leq 1.6 \ll Q_{\mathrm{soln}, 2}=15.5$. According to the analysis given in section 3 (cf. (3.24)), the overall quality of the resulting adaptive meshes is good.

Example 6.2. The second example is

$$
v(x, y)=e^{-\frac{x}{\epsilon}}+e^{-\frac{y}{\epsilon}}, \quad(x, y) \in \Omega=(0,1) \times(0,1),
$$

where $\epsilon=0.01$. It exhibits a strong anisotropic feature - boundary layers along $x=0$ and $y=0$. This function was used in section 4 to illustrate the mesh quality measures. We use it here to show the ability of the bound-based and dimensional balancing adaptation functionals to generate adaptive meshes of good quality.

The results obtained are shown in Figures 6.3 and 6.4 and Table 6.2. Similar observations can be made as for the previous example, except that $\left\|Q_{\text {geo }}\right\|_{\infty}$ is large (around 24) for the current example, indicating that the adaptive mesh has long, thin
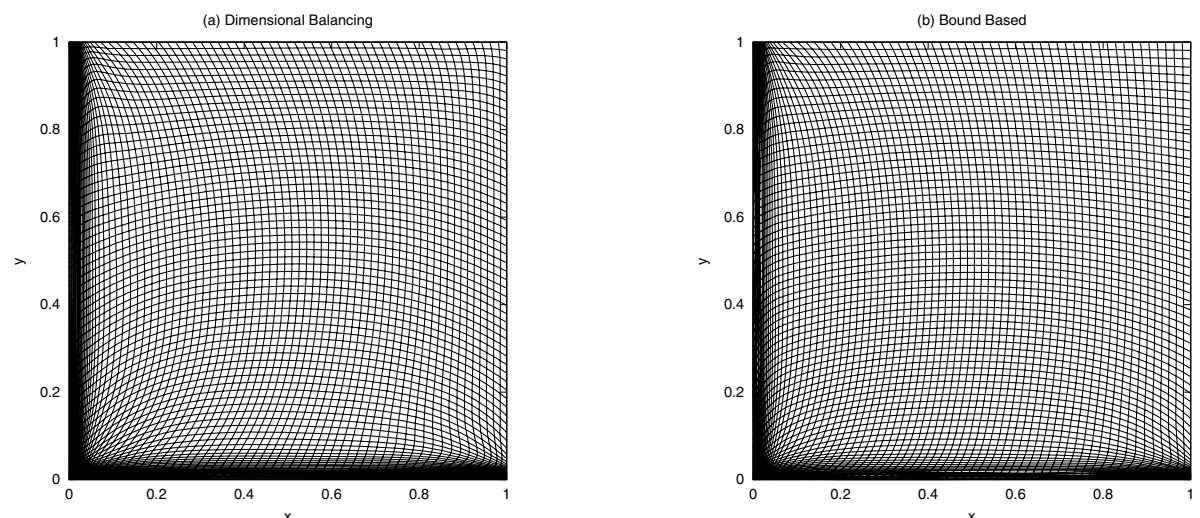

FIG. 6.3. Adaptive meshes of size $81 \times 81$ are obtained for Example 6.2 with the bound-based and dimensional balancing functionals. 

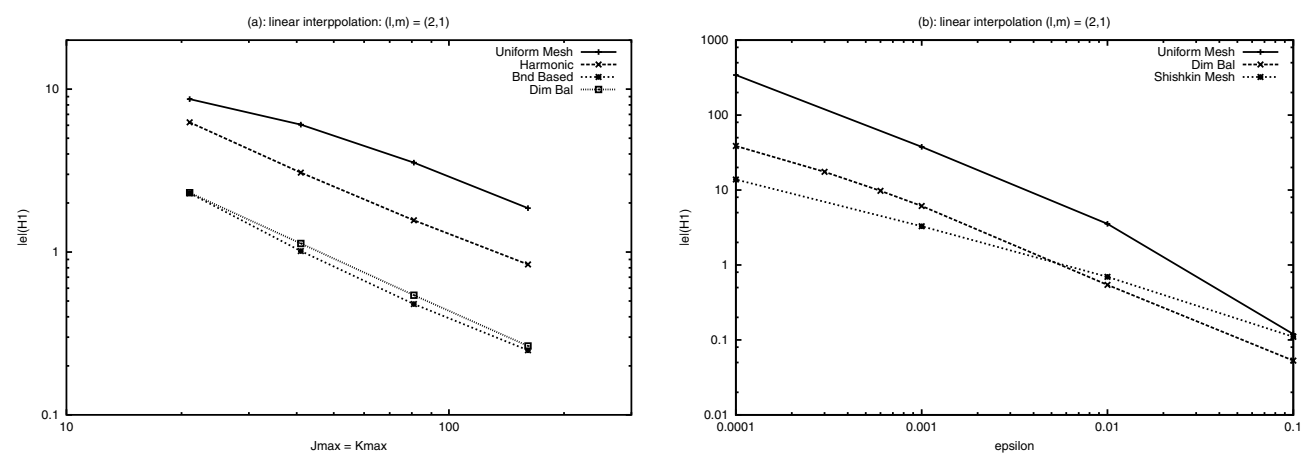

FIG. 6.4. Example 6.2: (a) The linear interpolation error $|e|_{H^{1}}$ is plotted as a function of Jmax. (b) The error $|e|_{H^{1}}$ obtained on a mesh of size $81 \times 81$ is plotted as a function of $\epsilon$.

TABLE 6.2

Quality measures for meshes obtained for Example 6.2 with the bound-based (Bnd-based) and dimensional balancing (Dim bal) functionals.

\begin{tabular}{l|c|c|c|c|cc}
\hline \hline Method & $J \max$ & $\left\|Q_{\text {geo }}\right\|_{\infty}$ & $\left\|Q_{\text {ali, } 2}\right\|_{\infty}$ & $\left\|Q_{\text {adp }, 2}\right\|_{\infty}$ & $Q_{\text {mesh }, 2}$ & $Q_{\text {soln }, 2}$ \\
\hline \hline Dim bal & 21 & 14.7 & 3.32 & 2.41 & 2.66 & 145.5 \\
& 41 & 18.9 & 4.56 & 2.67 & 2.96 & 145.5 \\
& 81 & 20.8 & 4.94 & 2.78 & 2.99 & 145.5 \\
& 161 & 23.1 & 5.19 & 2.80 & 2.97 & 145.5 \\
\hline \hline Bnd-based & 21 & 17.6 & 2.48 & 2.63 & 2.70 & 145.5 \\
& 41 & 22.4 & 3.88 & 2.07 & 2.73 & 145.5 \\
& 81 & 24.8 & 3.97 & 1.99 & 2.66 & 145.5 \\
& 161 & 13.7 & 2.47 & 3.21 & 2.69 & 145.5 \\
\hline \hline
\end{tabular}

elements. Nevertheless, the overall mesh quality measure remains small: $Q_{\text {mesh,2 }} \leq$ $3 \ll Q_{\text {soln }}=145.5$. According to the analysis in section 3 , the overall quality of the resulting meshes is good. In addition, Figure 6.4(a) shows that the interpolation error on an adaptive mesh generated using the bound-based or dimensional balancing functional is significantly smaller than that on a uniform mesh or an adaptive mesh generated using the harmonic map method.

Finally, Figure 6.4(b) shows the error $|e|_{H^{1}(\Omega)}$ as a function of $\epsilon$ for uniform, Shishkin-type, and adaptive meshes. It can be seen that the errors with the Shishkintype and adaptive meshes are significantly smaller than that on a uniform mesh for small $\epsilon$. The error on the adaptive mesh is slightly larger than that on the Shishkintype mesh for $\epsilon<4 \times 10^{-3}$. The dependence of the error on $\epsilon$ is $|e|_{H^{1}(\Omega)}=O\left(\epsilon^{-1}\right)$ for the uniform mesh, $O\left(\epsilon^{-\frac{1}{2}}|\ln \epsilon|^{2}\right)$ for the adaptive mesh, and $O\left(\epsilon^{-\frac{1}{2}}|\ln \epsilon|\right)$ for the Shishkin-type mesh.

7. Conclusions. In the previous sections we have studied mesh assessment based on the interpolation error estimates (2.10) and (2.13). According to these estimates, three elementwise measures were introduced: the geometric, alignment, and adaptation quality measures. The overall mesh quality measure, $Q_{\text {mesh }}$, is defined as the weighted $L^{q}$ norm of a product of the three elementwise quality measures; see (3.18) and (3.41). This definition takes into account the shape and size of elements and the solution behavior. In particular, the inclusion of equidistribution or adaptation renders the overall mesh quality measure suitable for assessing adaptive meshes and 
makes it relatively straightforward to incorporate it into a mesh adaptation strategy. It also makes the measure distinct from existing ones [3].

The effects of element shape and size and their interaction with the solution can be understood from the definition of $Q_{\text {mesh }}$. Indeed, the geometry, alignment, and adaptation mesh qualities directly affect the overall mesh quality and thus the interpolation error. In the meantime, their effects also compensate for each other since the three measures appear in $Q_{\text {mesh }}$ as a product. As a consequence, a mesh can maintain a good overall quality when its small elements (with a small adaptation measure) are shaped worse in the sense of having a large geometric measure than large elements. It was shown in section 3 that the overall quality of a mesh is good if $Q_{\text {mesh }}$ is small or significantly smaller than the roughness measure of the solution (see (3.22) and (3.42)), which is defined as the ratio of two Lebesgue norms of a derivative of the solution.

The definition of $Q_{\text {mesh }}$ given in section 3 comes in such a way that the measure appears in the error bound as the only factor depending substantially on the mesh. As an immediate result, the task of mesh adaptation becomes controlling the overall mesh quality. This idea has been applied to variational mesh adaptation, and two adaptation functionals have been developed. It has been demonstrated numerically that these functionals are able to generate adaptive meshes of good quality.

\section{REFERENCES}

[1] R. A. Adams, Sobolev Spaces, Academic Press, New York, 1975.

[2] T. Apel, Anisotropic Finite Elements: Local Estimates and Applications, Adv. Numer. Math., B. G. Teubner Stuttgart, Liepzig, 1999.

[3] T. Apel, M. Berzins, P. K. Jimack, G. Kunert, A. Plaks, I. Tsukerman, and M. Walkley, Mesh shape and anisotropic elements: Theory and practice, in The Mathematics of Finite Elements and Applications X, J. R. Whiteman, ed., Elsevier, Oxford, U.K., 2000, pp. 367376.

[4] T. Apel and M. Dobrowolski, Anisotropic interpolation with applications to the finite element method, Computing, 47 (1992), pp. 277-293.

[5] T. Apel and G. Lube, Anisotropic mesh refinement for a singularly perturbed reactiondiffusion model problem, Appl. Numer. Math., 26 (1998), pp. 415-433.

[6] I. BABUS̆KA AND A. K. AzIz, On the angle condition in the finite element method, SIAM J. Numer. Anal., 13 (1976), pp. 214-226.

[7] M. BERZINS, A solution-based triangular and tetrahedral mesh quality indicator, SIAM J. Sci. Comput., 19 (1998), pp. 2051-2060.

[8] J. U. Brackbill, An adaptive grid with directional control, J. Comput. Phys., 108 (1993), pp. $38-50$.

[9] J. U. Brackbill and J. S. Saltzman, Adaptive zoning for singular problems in two dimensions, J. Comput. Phys., 46 (1982), pp. 342-368.

[10] H. G. Burchard, Splines (with optimal knots) are better, Appl. Anal., 3 (1974), pp. 309-319.

[11] P. G. Ciarlet, The Finite Element Method for Elliptic Problems, North-Holland, Amsterdam, 1978.

[12] V. Dolejsi, Anisotropic mesh adaptation for finite volume and finite element methods on triangular meshes, Computing and Visualisation in Science, 1 (1998), pp. 165-178.

[13] A. S. Dvinsky, Adaptive grid generation from harmonic maps on Riemannian manifolds, J. Comput. Phys., 95 (1991), pp. 450-476.

[14] J. E. Castillo, Ed., Mathematical Aspects of Numerical Grid Generation, Frontiers in Appl. Math. 8, SIAM, Philadelphia, 1991.

[15] R. HAGMEEIJER, Grid adaption based on modified anisotropic diffusion equations formulated in the parametric domain, J. Comput. Phys., 115 (1994), pp. 169-183.

[16] G. H. Hardy, J. E. Littlewood, and G. Pólya, Inequalities, Cambridge University Press, Cambridge, UK, 1934.

[17] W. Huang, Practical aspects of formulation and solution of moving mesh partial differential equations, J. Comput. Phys., 171 (2001), pp. 753-775. 
[18] W. HuAng, Variational mesh adaptation: Isotropy and equidistribution, J. Comput. Phys., 174 (2001), pp. 903-924.

[19] W. Huang And W. Sun, Variational mesh adaptation II: Error estimates and monitor functions, J. Comput. Phys., 184 (2003), pp. 619-648.

[20] P. Jamet, Estimations de l'erreur pour des elements finis droits preque degeneres, RAIRO Anal. Numer., 10 (1976), pp. 43-60.

[21] P. Knupp, Mesh generation using vector-fields, J. Comput. Phys., 119 (1995), pp. 142-148.

[22] P. Knupp and S. Steinberg, Fundamentals of Grid Generation, CRC Press, Boca Raton, FL, 1994.

[23] P. M. Knupp, Jacobian-weighted elliptic grid generation, SIAM J. Sci. Comput., 17 (1996), pp. $1475-1490$.

[24] P. M. KNupP AND N. RoBidoux, A framework for variational grid generation: Conditioning the Jacobian matrix with matrix norms, SIAM J. Sci. Comput., 21 (2000), pp. 2029-2047.

[25] H. Kober, On the arithmetic and geometric means and on Hölder's inequality, Proc. Amer. Math. Soc., 9 (1958), pp. 452-459.

[26] M. KŘÍžEK, On the maximum angle condition for linear tetrahedral elements, SIAM J. Numer. Anal., 29 (1992), pp. 513-520.

[27] G. Kunert, A Posteriori Error Estimation for Anisotropic Tetrahedral and Triangular Finite Element Meshes, Ph.D. thesis, Technische Universiti Chemnitz, Chemnitz, Germany, 1999.

[28] V. D. Liseikin, Grid Generation Methods, Springer, Berlin, 1999.

[29] S. Steinberg And P. J. Roache, Variational grid generation, Numer. Methods Partial Differential Equations, 2 (1986), pp. 71-96.

[30] J. L. Synge, The Hypercircle in Mathematical Physics, Cambridge University Press, Cambridge, UK, 1957.

[31] J. F. Thompson, Z. A. Warsi, And C. W. Mastin, Numerical Grid Generation: Foundations and Applications, North-Holland, New York, 1985.

[32] A. Winslow, Numerical solution of the quasi-linear Poisson equation in a nonuniform triangle mesh, J. Comput. Phys., 1 (1967), pp. 149-172.

[33] Z. Zhang And A. NaGa, A new finite element gradient recovery method, Part I: Superconvergence property, SIAM J. Sci. Comput., to appear.

[34] O. C. ZIENKIEWICZ AND J. Z. ZHU, The superconvergence patch recovery and a posteriori error estimates. Part 1: The recovery technique, Internat. J. Numer. Methods Engrg., 33 (1992), pp. 1331-1364.

[35] O. C. ZIENKIEWICZ AND J. Z. ZhU, The superconvergence patch recovery and a posteriori error estimates. Part 2: Error esimates and adaptivity, Internat. J. Numer. Methods Engrg., 33 (1992), pp. 1365-1382.

[36] M. Zlámal, On the finite element method, Numer. Math., 12 (1968), pp. 394-409. 